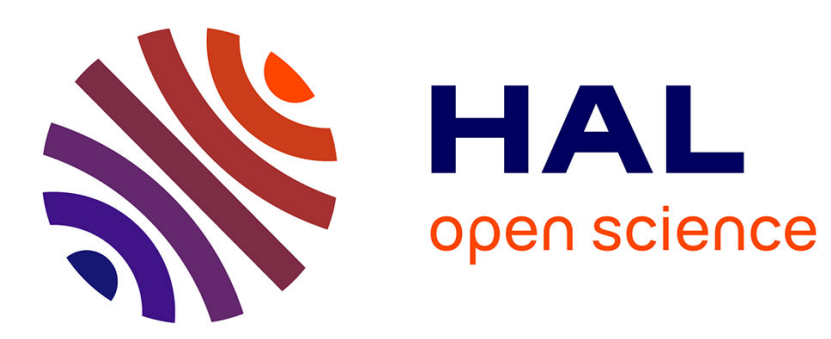

\title{
Magnetic Coupling in the Ce(III) Dimer Ce-2(COT)(3)
}

Frédéric Gendron, Jochen Autschbach, Jean-Paul Malrieu, Hélène Bolvin

\section{To cite this version:}

Frédéric Gendron, Jochen Autschbach, Jean-Paul Malrieu, Hélène Bolvin. Magnetic Coupling in the $\mathrm{Ce}(\mathrm{III})$ Dimer Ce-2(COT)(3). Inorganic Chemistry, 2019, 58 (1), pp.581-593. 10.1021/acs.inorgchem.8b02771 . hal-01997978

\section{HAL Id: hal-01997978 \\ https://hal-univ-rennes1.archives-ouvertes.fr/hal-01997978}

Submitted on 2 Dec 2020

HAL is a multi-disciplinary open access archive for the deposit and dissemination of scientific research documents, whether they are published or not. The documents may come from teaching and research institutions in France or abroad, or from public or private research centers.
L'archive ouverte pluridisciplinaire HAL, est destinée au dépôt et à la diffusion de documents scientifiques de niveau recherche, publiés ou non, émanant des établissements d'enseignement et de recherche français ou étrangers, des laboratoires publics ou privés. 


\title{
Magnetic Coupling in the Ce(III) Dimer $\mathrm{Ce}_{2}(\mathrm{COT})_{3}$
}

\author{
Frédéric Gendron $^{\mathrm{a} b}$, Jochen Autschbach ${ }^{\mathrm{a}}$, Jean-Paul Malrieu ${ }^{\mathrm{c}}$, Hélène Bolvin $^{\mathrm{c} d *}$,
}

Dedicated to Jean-Paul Malrieu on the occasion of his 80th birthday, for his contribution to the modelling and the understanding of magnetic properties.

\footnotetext{
Abstract

The monomer $\left[\mathrm{Ce}(\mathrm{COT})_{2}\right]^{-}$and the dimer $\left[\mathrm{Ce}_{2}(\mathrm{COT})_{3}\right]$, with $\mathrm{Ce}(\mathrm{III})$ and $\mathrm{COT}=1,3,5,7-$ cyclooctatetraenide, are studied by quantum chemistry calculations. Due to the large spinorbit coupling, the ground state of the monomer is a strong mixing of $\sigma$ and $\pi$ states. The experimental isotropic coupling in the dimer was evaluated by Walter et al. to be $J=-7 \mathrm{~cm}^{-1}$ (with a Heisenberg Hamiltonian $\hat{\mathcal{H}}_{S}=-J \hat{S}^{A} \cdot \hat{S}^{B}$ ) with a small anisotropic coupling of $0.02 \mathrm{~cm}^{-1}$. The coupling between the two Ce(III) in the dimer is calculated using CI methods. The low energy part of the spectra are modeled by spin Hamiltonians. All spin Hamiltonians parameters are deduced from $a b$ initio calculations. $g$ factors are calculated for both the pseudo-doublet of the monomer and the pseudo-triplet of the dimer and their sign have been determined. The magnetic coupling in the dimer is rationalized by a model based on crystal field theory. The kinetic and exchange contributions arising from the different configurations to the isotropic and anisotropic couplings are evaluated. It is shown

a Department of Chemistry, University at Buffalo, State University of New York, Buffalo, New York 142603000, United States

bPresent address: Univ. Rennes ISCR (Institut des Sciences Chimiques de Rennes) UMR 622635042 Rennes, France

${ }^{c}$ Laboratoire de Chimie et Physique Quantiques, CNRS, Université Toulouse III, 118 route de Narbonne, 31062 Toulouse, France

${ }^{\mathrm{d}}$ On research leave at Hylleraas Center for Quantum Molecular Sciences, Department of Chemistry, University of Oslo, Norway for the period August 2017 to June 2018.

*e-mail address: bolvin@irsamc.ups-tlse.fr
}

that the main contribution to isotropic coupling is kinetic and originates from the $f_{\sigma}-f_{\sigma}$ interaction due to the large transfer integral between those orbitals. However the $f_{\pi}-f_{\pi}$ interaction plays a non-negligible role. The anisotropic coupling originates from the difference of exchange energy of states arising from the $f_{\sigma} f_{\pi}$ configuration and is, in no matter, related to the anisotropy of the local magnetic moments as already pointed by Van Vleck for a fictitious $s-p$ system. The analysis of the Natural Orbitals evidences a superexchange mechanism through a $\sigma_{C H}^{*}$ orbital of the bridging cycle favored by a local $4 f_{\sigma} / 5 d_{\sigma}$ hybridization and that the $\delta$ type orbitals, both the HOMOs of the ligands and the virtual $f_{\delta}$ orbitals of the cerium atoms play an important polarization role, and to a less extend the $\pi$ type orbitals, the HOMOs-1 of the ligands and the metal $f_{\pi}$ orbitals.

\section{Introduction}

Lanthanide complexes get more and more interest in molecular magnetism due to their properties of Single Molecule Magnets. ${ }^{1,2,3}$ Thanks to a large orbital contribution, they present a large magnetic moment and a strong anisotropy. In order to increase the performance of these properties, one should optimize the coupling between the metal centers in order to build large magnetic clusters. Indeed, the magnetic properties of lanthanides are borne by the $4 f$ orbitals which have a semi-internal character; their interaction with the environment is extremely weak, this leads to the non quenching of the orbital moment and to a large magnetic anisotropy but this makes the coupling between magnetic sites being tiny. Designing binuclear lanthanide-containing complexes with sizable exchange coupling is a challenge. The study of the interplay of the Single Molecule Magnets behavior in binuclear complexes is quite 
recent and is mainly focused on Dy-containing complexes. $^{4,5,6,7,8,9,10,11,12,13,14}$ A better knowledge of the microscopic mechanisms is necessary and for this point, theoretical tools can bring essential elements. The theoretical description of the magnetic coupling between two lanthanide centers is usually performed through a fitting procedure and to our knowledge, there has been no first principle description of this coupling. The aim of this article is to present an extensive analysis of the coupling between two cerium (III) ions using first principle quantum chemistry tools.

Van Vleck and Dirac pointed out long ago that the 'magnetic' interaction between two electrons, namely the difference of energy between the singlet and the triplet states, is formally equivalent to the 'cosine coupling' between the spin magnetization operators $\hat{\boldsymbol{s}}_{1}$ and $\hat{\boldsymbol{s}}_{2}$ and that the interaction between the two electrons may be described by the following Hamiltonian

$$
\hat{\mathcal{H}}^{12}=-J \hat{\boldsymbol{s}}^{1} \cdot \hat{\boldsymbol{s}}^{2}
$$

where $J$ is the exchange integral ${ }^{15,16}$ between the singly occupied orbitals as previously derived by Dirac ${ }^{17}$ The underlying interaction is nothing magnetic since it arises from Pauli principle and the antisymmetrization of the wave functions; it is not an interaction between the spin magnetic moments but it depends on the orbitals $i$ which the electrons are by the mean of $J$. Eq. 1 is still valid to describe the exchange between two atoms $A$ and $B$ with half filled shells as the interaction between the two local total spins $\boldsymbol{S}_{A}$ and $S_{B}$ by replacing $J$ by the sum over the different exchange integrals between all open shell electrons of $A$ and $B^{18}$

$$
\hat{\mathcal{H}}^{A B}=-J \hat{S}^{A} \cdot \hat{S}^{B}
$$

This so called Heisenberg-Dirac-van Vleck Hamiltonian is used phenomenologically and $J$ is a phenomenological parameter taking into account the so called antiferromagnetic kinetic exchange due to the charge transfer between the two sites. ${ }^{19}$

Van Vleck showed ${ }^{18}$ that the exchange interaction between two atoms $A$ and $B$ with one $p$ and one $s$ electron respectively is anisotropic and may be described in terms of the total angular momentum $\hat{\boldsymbol{j}}^{A}$ of the $p$ electron and the spin $\hat{\boldsymbol{s}}^{B}$ of the $s$ electron, within the ${ }^{2} P_{1 / 2} \otimes^{2} \Sigma$ manifold,

$$
\begin{aligned}
\hat{\mathcal{H}}^{A B} & =-J \hat{\boldsymbol{j}}^{A} \cdot \hat{\boldsymbol{s}}^{B}+\hat{\boldsymbol{j}}^{A} \cdot \mathbf{D} \cdot \hat{\boldsymbol{s}}^{B} \\
& =-J \hat{\boldsymbol{j}}^{A} \cdot \hat{\boldsymbol{s}}^{B}+D\left[3 \hat{j}_{z}^{A} \hat{s}_{z}^{B}-\hat{\boldsymbol{j}}^{A} \cdot \hat{\boldsymbol{s}}^{B}\right]
\end{aligned}
$$

with $J=\frac{2}{9}\left(2 K_{\Pi}+K_{\Sigma}\right)$ and $D=\frac{4}{9}\left(K_{\Pi}-K_{\Sigma}\right)$, $K_{\Sigma}$ and $K_{\Pi}$ being the exchange integrals between $p_{z}^{A}, s^{B}$ and $p_{x, y}^{A}, s^{B}$ orbitals, respectively ( $z$ is the intermolecular axis). The anisotropy is described through $D$ and arises from the difference in exchange integrals $K_{\Sigma}$ and $K_{\Pi}$ through spin-orbit coupling (SOC) which mixes $p_{z}$ and $p_{x, y}$ orbitals. As pointed by Van Vleck "This example makes it clear that anisotropy in the exchange field can be generated without there being any anisotropy in the $g$ factors". $g$ factors refer here to the effective $g$-values of pseudo-spin states, as it will be the case all along this article.

In the case of lanthanide complexes, except for Gd(III), the $4 f$ shell is not half-filled, the orbital moment is not quenched and the magnetic moment of each site is anisotropic with large orbital contributions. The ground state of the free ion is determined by its total angular momentum value $\mathfrak{J}$ which $2 \mathfrak{J}+1$ degeneracy is removed by the effect of the ligands. In the free ion, the spin operator is related to the total angular momentum according to

$$
\hat{\boldsymbol{S}}=\left(g_{J}-1\right) \hat{\mathfrak{J}}
$$

where $g_{J}$ is the Landé $\mathrm{g}$ factor and Eq. 2 becomes $^{20}$

$$
\hat{\mathcal{H}}^{A B}=-J \hat{\mathfrak{J}}^{A} \cdot \hat{\mathfrak{J}}^{B}
$$

In this equation, $J$ comprises the $g$ factors and implicitly, its anisotropy is related to the one of the local $\mathrm{g}$ tensors. Although no justified a priori [21], this equation is useful since it needs only one parameter which might be determined by fitting experimental data; it is the case with Lines model, ${ }^{22}$ where the local properties are determined using $a b$ initio calculations and the interaction is modeled according to Eq. 5. This approach is valuable to calculate thermodynamic properties where the whole $2 \mathfrak{J}+1$ manifold of the free ion term is populated. The anisotropy of the coupling appears to be a consequence of the anisotropy of the local $\mathrm{g}$ tensors, or in other terms, of the local magnetic moments. Combined with a first principle description of the monomers, it permits to determine the value of $J$ either using a mean field 
approach as proposed by Chibotaru ${ }^{23}$ or by a state by state procedure. ${ }^{14}$ Eq. 5 is usually generalized as the interaction between two local $\hat{\mathfrak{J}}$ angular momenta and may be expressed using the irreducible tensor algebra $24,25,26,27,21$

$$
\hat{\mathcal{H}}^{A B}=\sum_{k q k^{\prime} q^{\prime}} J_{k q k^{\prime} q^{\prime}} \frac{O_{k}^{q}\left(\hat{\mathfrak{J}}^{A}\right) O_{k^{\prime}}^{q^{\prime}}\left(\hat{\mathfrak{J}}^{B}\right)}{O_{k}^{0}\left(\mathfrak{J}^{A}\right) O_{k^{\prime}}^{0}\left(\mathfrak{J}^{B}\right)}
$$

where the $O_{k}^{q}$ are Stevens operators and the parameters $J_{k q k^{\prime} q^{\prime}}$ are either considered as phenomenological parameters fitted on experimental data or may be calculated as effective parameters determined from larger model spaces including more configurations, as for examples the charge transfer states $4 f^{N-1}-4 f^{N+1}$ and the $5 d$ shell $4 f^{N-1}-4 f^{N} 5 d .^{28}$

A local Kramers doublet (KD) is represented by a pseudo-spin $\mathbb{S}^{A}=1 / 2$ and its anisotropic magnetic moment by $\mathbf{g}^{A} \cdot \hat{\mathbf{S}}^{A}$. Eq. 5 restricted to the interaction between two KDs may be rewritten as

$$
\hat{\mathcal{H}}^{A B}=J\left(\mathbf{g}^{A} \cdot \hat{\mathbb{S}}^{A}\right) \cdot\left(\mathbf{g}^{B} \cdot \hat{\mathbb{S}}^{B}\right)
$$

But, if one does not want to tie the anisotropy of the coupling to the one of the local moments, the most general Spin Hamiltonian describing two local KDs in an external magnetic field $\boldsymbol{B}$ takes the form

$$
\begin{aligned}
\hat{\mathcal{H}}_{S}= & \mu_{B} \boldsymbol{B} \cdot \mathbf{g}^{A} \cdot \hat{\mathbb{S}}^{A}+\mu_{B} \boldsymbol{B} \cdot \mathbf{g}^{B} \cdot \hat{\mathbf{S}}^{B} \\
& +J \hat{\mathbb{S}}^{A} \cdot \hat{\mathbb{S}}^{B}+\hat{\mathbb{S}}^{A} \cdot \mathbf{D} \cdot \hat{\mathbb{S}}^{B}
\end{aligned}
$$

where $\mathbf{D}$ is a traceless tensor. EPR permits to determine all the parameters of Eq. 8 as for example recently performed in the group of Chilton in $\mathrm{Dy}(\mathrm{III}), \mathrm{Er}(\mathrm{III})$ and $\mathrm{Yb}$ (III) dimers. $^{29,30}$

Sandwiches complexes formed with $\mathrm{COT}=1,3,5,7$-cyclooctatetraene have been extensively studied with different $\operatorname{Ln}(\mathrm{III})$ ions, both experimentally and theoretically, mostly with substituted forms of the ligands, ${ }^{31}, 32,33,34,35,36$ some of them presenting single-molecule magnet properties. ${ }^{37,38}$ The double decker $\left[\mathrm{Ce}(\mathrm{COT})_{2}\right]^{-}$and the triple decker $\left[\mathrm{Ce}_{2}(\mathrm{COT})_{3}\right]$ have been synthesized and their magnetic properties characterized by magnetometry and EPR by Walter et al.. ${ }^{33}$ These two complexes permit an accurate description by first principle calculations and a precise modelization due their high symmetry, very close to $\mathcal{D}_{8 h}$, and a simple electron configuration with one magnetic electron per center. This makes CI calculations feasible and reduces the models to a few number of parameters. Models for $\mathrm{Ce}(\mathrm{III})-\mathrm{Ce}(\mathrm{III})$ interactions (and $\mathrm{Yb}(\mathrm{III})-\mathrm{Yb}(\mathrm{III}) \mathrm{Cu}(\mathrm{III})-\mathrm{Cu}(\mathrm{III})$ ) have already been proposed. ${ }^{39,28,40}$

The aim of this work is to describe theoretically both $\left[\mathrm{Ce}(\mathrm{COT})_{2}\right]^{-}$and $\left[\mathrm{Ce}_{2}(\mathrm{COT})_{3}\right]$ The two complexes are first described using $a b$ initio methods and the calculations compared to available experimental data. For the monomer, as discussed in Section 3.2, the spin Hamiltonian of the ground KD, namely the $\mathbf{g}$ tensor is calculated and interpreted within a crystal field (CF) model presented in Section S4.1. For the dimer, as presented in Sections 3.3.1 and 3.3.2, the spin Hamiltonian of Eq. 8 is built from the two local KDs and then expressed with the total spin, a pseudo-singlet and a pseudo-triplet (see Section S4.2). Spin Hamiltonian parameters are deduced from $a b$ initio calculations. A model Hamiltonian, presented in Section S4.3, is proposed that permits to separate the coupling into kinetic and exchange contributions arising from each configuration. It is concluded that the anisotropy of the coupling is not related to the anisotropy of the local magnetic moments, but rather arises from the difference in exchange integrals between the states of configuration $f_{\sigma} f_{\pi}$, a mechanism closely related to the one pointed out by Van Vleck in Eq. 3 who wrote "We wish, as the main point of the present paper, to show that the anisotropy in the exchange field need not necessarily be the same as that in $g$ " and pointed out by Mironov. ${ }^{28}$ Finally, the orbitals involved in the coupling are analyzed; it evidences a superexchange mechanism through a $\sigma_{C H}^{*}$ orbital of the bridging cycle favored by a local $4 f_{\sigma} / 5 d_{\sigma}$ hybridization and that the $\delta$ type orbitals, both the HOMOs of the ligands and the virtual $f_{\delta}$ of the cerium atoms play an important polarization role, and to a less extend the $\pi$ type orbitals, the HOMOs-1 of the ligands and the metal $f_{\pi}$ orbitals. For better readability of the article, detailed technical derivations are presented in the Supplementary Material and briefly described in the main article. 


\section{Computational details}

\subsection{DFT calculations}

Structure optimizations and broken-symmetry calculations were performed using the 2014 release of the Amsterdam Density Functional (ADF) package p1, 42, 43 using the scalar relativistic (SR) all-electron zeroth-order regular approximation (ZORA) Hamiltonian ${ }^{44}$ and triple- $\zeta$ polarized all-electron Slater type basis (TZP) from the ADF basis-set library. Structure optimizations were performed with B3LYP functional ${ }^{45}$ within a spin doublet state for $\left[\mathrm{Ce}(\mathrm{COT})_{2}\right]^{-}$and a spin triplet state using an average of configurations that corresponds to distribute the two unpaired electrons into the $4 f$ orbitals of the two cerium centers spanning the $a_{2 u}$ and $e_{1 u}$ irreducible representations of the $D_{8 h}$ symmetry point group. Single point calculations were performed with either the non-hybrid generalized gradient approximation (GGA) functional $\mathrm{PBE}^{46,47}$ (Perdew-Burke-Ernzerhof), a hybrid version of PBE (PBE0) ${ }^{48}, 49$ with $25 \%$ of exact HartreeFock exchange $(\mathrm{eX})$, or the hybrid functional B3LYP $^{45}$ (Becke, three-parameter Lee-YangParr) with $20 \%$ eX. All calculations were performed within the $C_{8 v}$ symmetry using the spin-unrestricted approach. The energies of the antiferromagnetic states were obtained using broken-symmetry approach developed by Noodleman. ${ }^{50}$ Scalar relativistic spin densities were plotted with the ADF-GUI package.

\subsection{WFT calculations}

Wave Function Theory (WFT) calculations were performed using MOLCAS-78 suite of programs. $^{51}$ ANO-RCC basis sets with TZP quality were used on all atoms. ${ }^{52,53}$ The active space consisted of the $4 f$ electrons in the $4 f$ orbitals: $\operatorname{CAS}(1,7)$ for $\left[\mathrm{Ce}(\mathrm{COT})_{2}\right]^{-}$ and $\operatorname{CAS}(2,14)$ for $\left[\mathrm{Ce}_{2}(\mathrm{COT})_{3}\right]$. For the monomer, the active space has been extended to $\operatorname{RAS}(9 / 7 / 4)$ with up to 2 holes in RAS1 (which comprises the five $4 d$ of the metal and the four highest occupied $\pi$ orbitals of the ligands) and up to two particles in RAS3 (which comprises the four lowest unoccupied $\pi$ of the ligands). First a multi-state SF-CASSCF (SpinFree Complete Active Space Self Consistent Field $)^{54}$ calculation was performed with the seven doublet states. Dynamical correlation may be included at second order of perturbation theory using CASPT2 method. ${ }^{55}$ SOC was included by a state interaction between the CASSCF or the MS-CASPT2 wave functions by RASSI (Restricted Active Space State Interaction) method $^{56}$; one gets the SO-CASSCF and SO-CASPT2 results respectively. 7 doublets for the monomer and 49 triplets and 49 singlets for the dimer were considered for the calculation of the SOC. Scalar relativistic effects were taken into account by means of Douglas-KrollHess transformation [57] and Spin-Orbit (SO) integrals are calculated using the AMFI (Atomic Mean-Field Integrals) approximation. ${ }^{[58]} \mathrm{Calcu}-$ lations were performed without considering the symmetry for the monomer and within the $\mathcal{C}_{i}$ point group for the dimer. Spin Hamiltonian parameters were deduced from RASSI calculations; from the matrix elements of the magnetic moment operator $\hat{\boldsymbol{M}}=\hat{\boldsymbol{L}}+g_{e} \hat{\boldsymbol{S}},{ }^{59,60}$ for the monomer following ${ }^{61}$ and for the dimer as summarized in Section S4.2. Geometry optimizations were performed by optimizing only the Cering distances with a frozen geometry for the rings ; the radius of the ring $\mathrm{r}_{C O T}=1.831 \AA$ and $r_{C H}=1.031 \AA$.

Configuration Interaction (CI) calculations were performed with the CASDI program using the $\mathcal{D}_{2 h}$ symmetry [62]. The DDCIn (Difference Dedicated Configuration Interaction) method is variational: ${ }^{63}$ it considers the space formed following excitations out of the CAS; if $S_{1}\left(S_{3}\right)$ is the space of the orbitals which are doubly occupied (unoccupied) in the CASCI (Complete Active Space Configuration Interaction) calculation and $n_{h}\left(n_{p}\right)$ the number of allowed holes (particles) in $S_{1}\left(S_{3}\right)$, the DDCIn space contains all the configurations satisfying $n_{h}+n_{p} \leq n$. CI calculations involved 110 electrons in 375 orbitals. CAS $+\mathrm{S}$ CI corresponds to $n_{h} \leq 1$ and $n_{p} \leq 1$. Two active spaces have been considered: $\operatorname{CAS}(2,6)$ with $f_{\sigma}$ and $f_{\pi}$ orbitals for the $f_{\sigma} f_{\sigma}$, $f_{\sigma} f_{\pi}$ and $f_{\pi} f_{\pi}$ configurations up to DDCI3 and $\mathrm{CAS}(2,14)$ including all $f$ orbitals for other configurations, up to DDCI2. The ${ }^{3} B_{1 u}$ orbitals of the corresponding CASSCF calculation (with 3 and 10 roots respectively) were used. SO-XX (with XX=CASCI, CAS+S CI, DDCI2, DDCI3) results were obtained by dressing the $\mathrm{SO}$ matrix with XX energies. 


\section{Results and Discussion}

\subsection{Structural parameters}

The structure of the $\left[\mathrm{Ce}(\mathrm{COT})_{2}\right]^{-}$anion has been determined by $\mathrm{X}$-ray with either an ether coordinated potassium cation $\left[\mathrm{K}\left(\left(\mathrm{CH}_{3} \mathrm{OCH}_{2} \mathrm{CH}_{2}\right)_{2} \mathrm{O}\right)\right]^{+}{ }^{64}$ or with $\left[\mathrm{Na}(\text { thf })_{3}\right]^{+}$or $\left[\mathrm{Li}(\text { thf })_{4}\right]^{+} 65$ as counterions. With the two former ones, one of the $\mathrm{COT}^{2-}$ ligand bridges the cerium and the alkaline cation while with the latter, $\left[\mathrm{Ce}(\mathrm{COT})_{2}\right]^{-}$is separated from the counterion. In $\left[\mathrm{Li}(\mathrm{THF})_{4} \mathrm{Ce}(\mathrm{COT})_{2}\right]$, the $\left[\mathrm{Ce}(\mathrm{COT})_{2}\right]^{-}$entity is slightly distorted from $\mathcal{D}_{8 h}$ symmetry; the Ce-C length ranges between 2.719 and $2.791 \AA$, and the distance between the $\mathrm{Ce}$ atom and the two centers of the rings are 2.032 and $2.099 \AA$. In the following, we will consider averaged distances. Since no counterion is considered, geometry optimizations provide symmetrical structures. Optimized distances are summarized in Table 1 and compared to the experimental data. With CASPT2, only the distance between $\mathrm{Ce}$ and the rings has been optimized, the geometry of the ligands being kept fixed. CASPT2 underestimates the Ce-ring distance by $0.043 \AA$ while B3LYP overestimates it by $0.086 \AA$; the GGA PBE and hybrid PBE0 give results closer to the experimental values. ${ }^{66}$

There is no crystallographic data for $\left[\mathrm{Ce}_{2}(\mathrm{COT})_{3}\right]$ dimer but $L_{I I I}$-edge EXAFS shows that Ce-Ce distance is $4.04 \AA$ and the Ce-C ones are 2.68 and $2.78 \AA$ for the outer and inner rings respectively. ${ }^{33}$ This leads to a C-C distance of $1.462 \AA$, much larger than both the $\mathrm{X}$-rays and B3LYP ones for the monomer. The complex has been fully optimized with B3LYP and Ce-ring distances have been optimized with CASPT2 with frozen ligands. In all cases, the outer ring is closer to $\mathrm{Ce}$ than the inner one since the latter is more loosely bound, being interacting with two $\mathrm{Ce}$ ions, but the effect is overestimated $(0.28 \AA)$ in geometry optimizations compared to $\operatorname{EXAFS}(0.1 \AA)$. The optimized Ce- $\mathrm{C}_{\text {out }}$ are on both sides of the EXAFS one but the optimized Ce- $\mathrm{C}_{\text {int }}$ are both longer than the EXAFS one. In the following of this work, three geometries are considered: the fully optimized B3LYP, and CASPT2 and EXAFS ones with the geometry of the cycle issued from X-rays in the mononuclear complex. For the so-called EXAFS structure, this choice leads to a longer Ce-Ce distance than the one predicted by Walter. The Ce-Ce distance between the two centers varies from 4.577 to $4.184 \AA$; this distance plays a key role in the magnetic coupling and studying those three geometries permit to analyze the effect of this distance.

\section{$3.2\left[\mathrm{Ce}(\mathrm{COT})_{2}\right]^{-}$}

In pseudo-axial $\mathcal{D}_{8 h}$ symmetry, $M_{J}$ behaves as a good quantum number ${ }^{67}$ and the ground term of the $4 f^{1}$ free ion ${ }^{2} F_{5 / 2}$ splits into three KDs $M_{J}= \pm 1 / 2, \pm 3 / 2, \pm 5 / 2$ due to the field of the ligands, the $M_{J}= \pm 1 / 2$ doublet being the ground state. The composition of the ground state may be modeled within a $\mathrm{CF}$ approach following the lines of Gourier for $\left[\mathrm{U}\left(\eta^{7}-\mathrm{C}_{7} \mathrm{H}_{7}\right)_{2}\right]^{-68}$ and Notter et al. for the $\mathrm{AnX}_{6}$ series, ${ }^{69}$ as summarized in $\mathrm{S} 4.1{ }^{70}$ The ground doublet arises for $99 \%$ from ${ }^{2} F_{5 / 2}$ with a small mixing of $1 \%$ with ${ }^{2} F_{7 / 2}$ due to CF. Within another perspective, it results from the mixing of $f_{\sigma}$ with $f_{\pi}$ spin orbitals with opposite spin by SOC

$$
\begin{aligned}
|\mathcal{D},+\rangle & =a f_{\sigma}-b \bar{f}_{\pi+} \\
|\mathcal{D},-\rangle & =\mathcal{K}|\mathcal{D},+\rangle=a \bar{f}_{\sigma}-b f_{\pi-}
\end{aligned}
$$

with $a^{2}=0.54, b^{2}=0.46, \pi_{ \pm}$correspond to $m_{l}= \pm 1$ and $\mathcal{K}$ the time reversal operator. $g$ factors are very sensitive to this mixing.

Magnetic properties of $\left[\mathrm{Li}(\text { thf })_{4}\right]\left[\mathrm{Ce}(\mathrm{COT})_{2}\right]$ have been characterized using different spectroscopies. The structure has been determined with X-ray diffraction and EXAFS, magnetic susceptibility recorded with a SQUID within the 5-300 $\mathrm{K}$ temperature range, revealing a TIP (Temperature Independent Paramagnet) behavior and the nature of the ground state probed by EPR spectroscopy, confirming a $\left|M_{J}\right|=1 / 2$ doublet with $g_{\|}=2.272$ and $g_{\perp}=1.123$ [67].

Results from $a b$ initio calculations on $\left[\mathrm{Ce}(\mathrm{COT})_{2}\right]^{-}$are summarized in Table 2 and S1. At SF level, orbitals are denoted according to their value of $\left|m_{l}\right|$ (using $f_{\sigma}, f_{\pi}, f_{\delta}$ and $f_{\phi}$ notations) and spinors by their value of $\left|m_{j}\right|$. The ordering of the SF orbitals is shown in Figure 1 and has been analyzed in details in reference 71 . It was shown that, within the $\mathrm{CF}$ theory perspective, the quadrupolar contribution of $\left[\mathrm{Ce}(\mathrm{COT})_{2}\right]^{-}$complex may be seen as an oblate structure due the inner electron ring of the ligands and this leads to the stabilization 
Table 1: Bond lengths $(\AA)$ in $\left[\mathrm{Ce}(\mathrm{COT})_{2}\right]^{-}$and $\left[\mathrm{Ce}_{2}(\mathrm{COT})_{3}\right]$ from experiment and geometry optimizations. ring denotes the center of a $\left[\mathrm{Ce}_{2}(\mathrm{COT})_{3}\right]$ cycle, ext and int denote the outer and inner rings respectively.

\begin{tabular}{|c|c|c|c|c|c|c|}
\hline & \multicolumn{3}{|c|}{$\left[\mathrm{Ce}(\mathrm{COT})_{2}\right]^{-}$} & \multicolumn{3}{|c|}{$\left[\mathrm{Ce}_{2}(\mathrm{COT})_{3}\right]$} \\
\hline & $X$-rays $^{a}$ & CASPT2 & $B 3 L Y P$ & EXAFS $^{d}$ & CASPT2 & B3LYP \\
\hline Ce-ring & 2.043 & 2.000 & 2.129 & $1.957^{e}$ & 1.897 & 2.000 \\
\hline $\mathrm{Ce}-\mathrm{C}_{\text {out }}$ & $2.743^{b}$ & 2.712 & 2.814 & 2.68 & 2.636 & 2.717 \\
\hline C-C $\mathrm{C}_{\text {out }}$ & $1.401^{b}$ & $1.401^{c}$ & 1.408 & 1.401 & $1.401^{c}$ & 1.408 \\
\hline Ce-ring ${ }_{\text {int }}$ & & & & $2.092^{e}$ & 2.173 & 2.289 \\
\hline $\mathrm{Ce}-\mathrm{C}_{\text {int }}$ & & & & 2.78 & 2.842 & 2.937 \\
\hline C-C $\mathrm{C}_{\text {int }}$ & & & & 1.401 & $1.401^{c}$ & 1.408 \\
\hline $\mathrm{Ce}-\mathrm{Ce}$ & & & & 4.184 & 4.346 & 4.577 \\
\hline
\end{tabular}

$a$ : from [65] in $\mathrm{Ce}(\mathrm{COT})_{2} \operatorname{Li}(\mathrm{thf}) ; b$ : averaged; $c$ : not optimized. $d$ : from [33] $e$ : supposing $d_{C-C}=1.401 \AA$

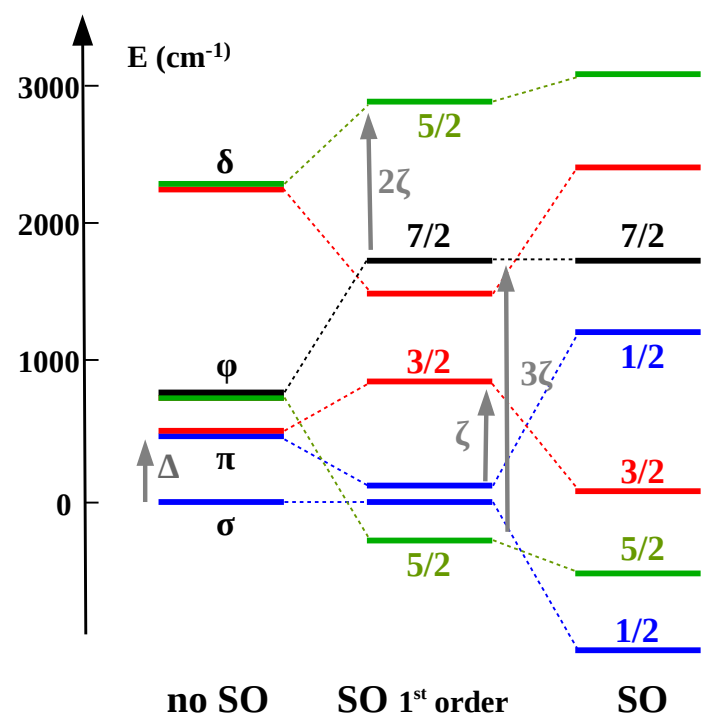

Figure 1: $4 f$ spinors scheme for $\left[\mathrm{Ce}(\mathrm{COT})_{2}\right]^{-}$, without SOC, with 1st order SOC and with SOC. Spinors are labeled according to the value of $\left|m_{J}\right|$. $\Delta$ and $\zeta$ are $\mathrm{CF}$ and SOC parameters.

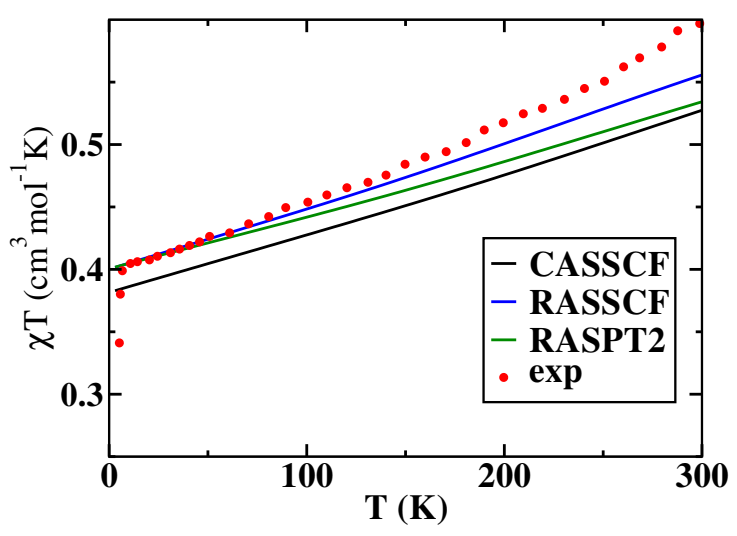

Figure 2: $\chi T$ vs $T$ for $\left[\mathrm{Ce}(\mathrm{COT})_{2}\right]^{-}$, experimental from [33] and calculated for X-rays geometry. $\mathrm{CAS}=\mathrm{CAS}(1,7) ; \mathrm{RAS}=\operatorname{RAS}(9 / 7 / 4)$

of $\sigma$ and $\pi$ orbitals. In addition, the $f_{\delta}$ orbitals are acceptors for weak ligand to metal donation bonding from ungerade combinations of the ligand HOMOs. Accordingly, there are occupied ligand-centered MOs with some ligand- $f_{\delta}$ bonding character, and unoccupied metal-centered MOs with antibonding character. Due to the latter, the $f_{\delta}$ are by far the most destabilized among the $4 f$ orbitals. First order SOC splits the degenerate orbitals according to their value of $\left|m_{j}\right|$, and then spinors with the same value of $\left|m_{j}\right|$ interact through 2 nd order SOC. The three KDs arising from $j=5 / 2$ are the lowest as they are in the free ion; the $\left|m_{j}\right|=1 / 2$ is the ground $\mathrm{KD}$ since it arises from the low lying $f_{\sigma}$ and $f_{\pi}$ orbitals, then the $\left|m_{j}\right|=5 / 2(\delta / \phi$ mixing $)$ and finally the $\left|m_{j}\right|=3 / 2(\pi / \delta$ mixing). The ordering of the $\left|m_{j}\right|$ follows the $f_{\left|m_{l}\right|}$ one and the reversal between $3 / 2$ and $5 / 2$ spinors arises from the large destabilization of the $f_{\delta} \cdot{ }^{71}$ 
Table 2: SO-CASSCF results for $\left[\mathrm{Ce}(\mathrm{COT})_{2}\right]^{-}$at geometries of Table 1. Ce-ring distance $d$ in $\mathrm{A}, \mathrm{SF}$ $\sigma-\pi$ gap $\Delta$ in $\mathrm{cm}^{-1}$, SOC parameter $\zeta$ in $\mathrm{cm}^{-1}$, $x=\zeta / \Delta$, coefficients of the wave function from Eq. $9(a, b)$ and from Eq. S6 $(A$ and $B)$ and $g$ factors.

\begin{tabular}{ccccc}
\hline geom & CASPT2 & X-rays & B3LYP & exp \\
\hline \hline$d$ & 2.000 & 2.043 & 2.129 & \\
$\Delta$ & 491 & 502 & 528 & \\
$\zeta$ & 667 & 669 & 672 & \\
$x$ & 0.736 & 0.751 & 0.786 & \\
$a$ & 0.731 & 0.732 & 0.736 & \\
$b$ & 0.683 & 0.681 & 0.677 & \\
$A$ & 0.994 & 0.994 & 0.994 & \\
$B$ & 0.105 & 0.108 & 0.113 & \\
$g_{\|}$ & 1.068 & 1.072 & 1.082 & 1.123 \\
$g_{\perp}$ & -2.388 & -2.382 & -2.370 & \pm 2.272 \\
\hline
\end{tabular}

CASPT2 underestimates the metal-ring distance while DFT overestimates it. The CF parameter $\Delta$ increases with $d$ which is not expected within neither a pure electrostatics nor a pure covalent picture but arises from the entanglement of these two points of view. ${ }^{71}$ The SOC parameter increases with distance, due to the decrease of covalency and the better localization of the spin density on the metal. $x=\zeta / \Delta$ increases slightly and is around 0.75 and as shown in Figure $\mathrm{S} 3, g_{\perp}$ is negative and decreases in absolute value while $g_{\|}$is positive and increases. $A^{2}$, the weight of the wave function on the ${ }^{2} F_{5 / 2}$ component is close to 1 , while $a^{2}$ and $b^{2}$, the weights of the wave function on $\sigma$ and $\pi$ components are almost equal. As in the $\mathrm{AcX}_{6}$ series and in $\left[\mathrm{U}\left(\eta^{7}-\mathrm{C}_{7} \mathrm{H}_{7}\right)_{2}\right]^{-}$, we are closer from the free ion limit with the $m_{j}= \pm 1 / 2$ component of the ${ }^{2} F_{5 / 2}$ coupled with the $m_{j}= \pm 1 / 2$ of the ${ }^{2} F_{7 / 2}$ than from the non relativistic limit with the lonely electron in the unquenched $\sigma$ orbital. The $g$ values are rather similar for the three geometries and match well the experimental ones. The temperature dependences of the experimental and calculated $\chi T$ are shown in Figure 2: calculations with the small active space (with 1 electron in the $4 f$ orbitals) underestimates the magnetization. Results are slightly improved with a larger active space, which comprises all $\pi$ orbitals of the ligands, both occupied and virtual, and the occupied $4 d$. This lowers the energy of the antibonding $f_{\delta}$ orbitals, and consequently the $3 \mathrm{rd}$ KD of symmetry $3 / 2$ which couples in the $\perp$ direction with the ground $\mathrm{KD} ; \chi T$ increases due to larger 2nd order Zeeman interaction.
The Natural Spin Orbitals (NSOs) correspond to the natural orbitals issued from the spin magnetization for a given direction of the external magnetic field as defined in references 72, 70, 73. NSOs and spin and orbital magnetization densities issued from the RASSCF calculation are represented in Figure 3. The spinors of Eqs. 9 correspond to the magnetization axis along direction $Z$ and one deduces the spinors for directions $X$ and $Y$ by rotating the quantification axis: those spinors are the magnetic ones bearing the lonely electron. Along $Z$ direction ( $Z$ is perpendicular to the rings), $f_{\sigma}$ and $f_{\pi}$ orbitals bear the $\alpha$ and $\beta$ spin densities while in the transverse direction, one $f_{\pi}$ is $\alpha$ and the other one $\beta$. The next contributions arise from $f_{\delta}$ and the HOMOs of the COT rings $\pi_{2}$, with $\alpha$ density in the bonding orbital and $\beta$ in the anti-bonding counterpart. This spin polarization is twice larger for the transverse direction than for the axial one. As it is usually the case, both spin and orbital magnetizations form rings in the plane perpendicular to the applied field, the orbital magnetization spreads more on the ligands than the spin one and this spreading is larger for transverse directions of the field than in the axial one, since in this latter case, there is no ligand in the equatorial plane.

The value of $g$ depends very little on the level of calculation. For X-rays/CASSCF, $g_{\|}^{L}=$ $2\left\langle m_{Z}^{L}\right\rangle=0.92, g_{\perp}^{L}=2\left\langle m_{X / Y}^{L}\right\rangle=3.43, g_{\|}^{S}=$ $2 g_{e}\left\langle m_{Z}^{S}\right\rangle=0.14$ and $g_{\perp}^{S}=2 g_{e}\left\langle m_{\perp}^{S}\right\rangle=-1.07$. From CF model, one gets (see Section S4.13.2)

$$
\begin{aligned}
g_{\|}^{L} & =2\left\langle\mathcal{D},+\left|\hat{L}_{z}\right| \mathcal{D},+\right\rangle=2 b^{2} \\
g_{\|}^{S} & =4\left\langle\mathcal{D},+\left|\hat{S}_{z}\right| \mathcal{D},+\right\rangle=2\left(a^{2}-b^{2}\right) \\
g_{\perp}^{L} & =2 \operatorname{Re}\left\langle\mathcal{D},+\left|\hat{L}_{x}\right| \mathcal{D},-\right\rangle=-4 \sqrt{3} a b \\
g_{\perp}^{S} & =4 \operatorname{Re}\left\langle\mathcal{D},+\left|\hat{S}_{x}\right| \mathcal{D},-\right\rangle=2 a^{2}
\end{aligned}
$$

$g_{\|}^{L}$ arises only from the $f_{\pi} \cdot g_{\|}^{S}$ as confirmed by Figure 3 is small due to the compensation between the $\alpha$ contribution of the $f_{\sigma}$ and the $\beta$ one of the $f_{\pi}$ : it appears on $m_{Z}^{S}(\boldsymbol{r})$ which evidences an alternating of $\alpha$ and $\beta$ layers. In the transverse direction, spin and orbital contributions are opposite in sign, the former arising only from $f_{\sigma}$ orbital since the two $f_{\pi}$ have opposite spins and the latter originates from the coupling between $f_{\sigma}$ and $f_{\pi}$ orbitals ( $a b$ term). 


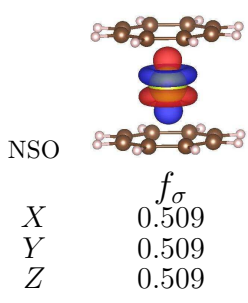

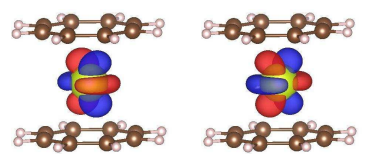

$f_{\pi}$

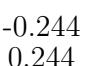

0.244

$-0.244$

0.244

$-0.244$

$-0.244$

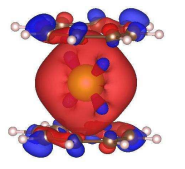

$m^{L}$

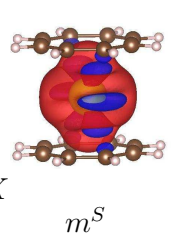

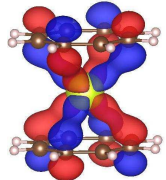

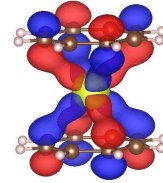

$f_{\delta}^{b}$

0.0021
0.0021

0.0021
0.0009

0.0021

0.0021

0.0009

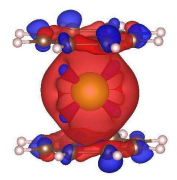

$m^{L}$
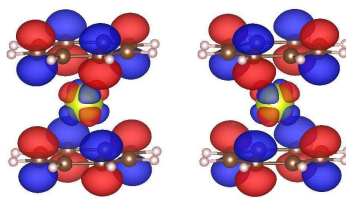

$f_{\delta}^{a b}$

$-0.0021$

$\begin{array}{ll}-0.0021 & -0.0021 \\ -0.0009 & -0.0009\end{array}$

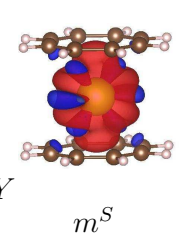

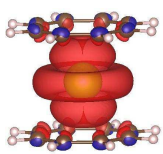

$m^{L}$

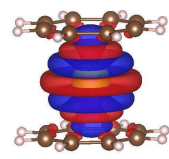

$m^{S}$

Figure 3: Isosurfaces and spin occupation of NSOs (top row) and isosurfaces of orbital and spin magnetizations (bottom row) for a magnetic field in directions $X, Y$ and $Z$ for the ground $\mathrm{KD}$ for $\left[\mathrm{Ce}(\mathrm{COT})_{2}\right]^{-}$ from RASSCF calculations with structure X-rays. Isovalues: \pm 0.03 au for orbitals and \pm 0.00001 for magnetization.

\section{$3.3\left[\mathrm{Ce}_{2}(\mathrm{COT})_{3}\right]$}

$\left[\mathrm{Ce}_{2}(\mathrm{COT})_{3}\right]$ has been synthesized and characterized by Walter et al. ${ }^{33}$ The two Ce(III) centers are in a $4 f^{1}$ configuration. Magnetic properties were characterized by SQUID magnetometry and EPR spectroscopy. The $\chi=f(T)$ curve as given in Figure 4 evidences an antiferromagnetic coupling between the two Ce(III) centers of $J=-7.9 \mathrm{~cm}^{-1}$ with a coupling Hamiltonian $\mathcal{H}=-J \mathbb{S}^{A} \cdot \mathbb{S}^{B}$ and $\mathbb{S}=1 / 2$ (this number is converted from reference 33 by a factor of -2 due to the change in the coupling Hamiltonian and from $\mathrm{K}$ to $\mathrm{cm}^{-1}$ ). The EPR spectrum has the characteristics of a spin triplet with $g_{\|}=0.974$ and $g_{\perp}=2.225$ and shows a zero-field splitting with $\left|D_{\|}\right|=0.020 \mathrm{~cm}^{-1}$ and $\left|D_{\perp}\right|=0.014 \mathrm{~cm}^{-1}$.

\subsubsection{DFT results}

The magnetic coupling in $\left[\mathrm{Ce}_{2}(\mathrm{COT})_{3}\right]$ has been calculated within the broken symmetry approach developed by Noodleman. ${ }^{50}$ The magnetic coupling constant within the $f_{\sigma}$ orbitals is deduced from the energies obtained with Kohn-Sham DFT for the scalar triplet state $\left|f_{\sigma}^{A} f_{\sigma}^{B}\right|$ and for the broken-symmetry solution $\left|f_{\sigma}^{A} \bar{f}_{\sigma}^{B}\right|$. As described by Noodleman, the broken-symmetry wave function represents a state of mixed spin with an energy $E_{B S}=$ $\frac{1}{2}\left(E_{\text {singlet }}+E_{\text {triplet }}\right)$. Therefore, the magnetic coupling constant $J$ in such can be deduced as

$$
J=2\left(E_{B S}-E_{T}\right)
$$

Due to the admixture of spin state, the brokensymmetry wave function often exhibits large spin contamination. In order to eliminate spin contamination, Yamagushi et al. formulated the following equation ${ }^{74}$

$$
J=2 \frac{E_{B S}-E_{T}}{\left\langle\hat{\boldsymbol{S}}^{2}\right\rangle_{T}-\left\langle\hat{\boldsymbol{S}}^{2}\right\rangle_{B S}}
$$

$\left\langle\hat{\boldsymbol{S}}^{2}\right\rangle_{(B S)}$ and $\left\langle\hat{\boldsymbol{S}}^{2}\right\rangle_{(T)}$ correspond to the square of the spin operator for both spin states and are generally admitted to be equal to 1.0 and 2.0 for systems containing two unpaired electrons. $\left\langle S^{2}\right\rangle$ values are given in Table S2. In all cases, $\left\langle\hat{\boldsymbol{S}}^{2}\right\rangle_{\mathrm{T}} \approx 2$ and $\left\langle\hat{\boldsymbol{S}}^{2}\right\rangle_{\mathrm{BS}} \approx 1$, confirming that we are in the strong localization limit and only Eq. 11 will be considered in the following. The magnetic coupling constants $J$ were calculated for the three geometries of Table 1 and are summarized in Table 3. The magnitude of $J$ depends on the choice of the functional, but in all cases the calculated $J$ remains negative, suggesting an antiferromagnetic coupling between the two cerium centers. The magnitude of the antiferromagnetic interaction increases with the shortening of the Ce-Ce distance. The GGA functional (PBE) gives the largest value of $J$, whereas the introduction of a percentage of exact Hartree-Fock exchange in PBE0 and B3LYP leads to a strong diminution of the calculated $J$ as it is usually the case for transition metal complexes. ${ }^{75}$ Overall, the KS-DFT results strongly overestimates the experimental magnetic coupling constant but it 
Table 3: Magnetic exchange coupling constants $J$ $\left(\mathrm{cm}^{-1}\right)$ calculated with SR DFT to Eq 11.

\begin{tabular}{lccc}
\hline Structure & B3LYP & PBE0 & PBE \\
\hline \hline EXAFS & -101.9 & -83.9 & -322.6 \\
CASPT2 & -53.7 & -43.9 & -160.6 \\
B3LYP & -21.7 & -17.3 & -83.8 \\
\hline
\end{tabular}

should be reminded that SOC is not included in those calculations, and it will be shown in next Section, that it plays a large reduction role.

\subsubsection{WFT results}

Excited states of $\left[\mathrm{Ce}_{2}(\mathrm{COT})_{3}\right]$ have been calculated with CAS based methods for the three geometrical structures of Table 1. Results are summarized in Table 4 and more details are given in Table S3. Without SOC, all states are easily identifiable according to a configuration $f_{m}^{A} f_{m^{\prime}}^{B}$. As expected, the two lowest states arise from the $f_{\sigma}^{A} f_{\sigma}^{B}$ configuration, leading to one spin singlet and one spin triplet. In all cases, the coupling is antiferromagnetic and it decreases rapidly with the distance between the two magnetic centers and increases with correlation. $\mathrm{CAS}+\mathrm{S}$ and DDCI2 give similar results. The DDCI3 couplings are about $2 / 3$ of the $J$ values obtained with hybrid functionals, which are the most reliable ones. As for DFT, the coupling is divided by a factor of 2 between EXAFS and PT2 structures while Ce-Ce distance decreases by $0.3 \AA$. The second manifold arises from $f_{\sigma}^{A} f_{\pi}^{B}$ configuration with an energy around the $\mathrm{CF}$ parameter $\Delta$ (ca. $\left.600 \mathrm{~cm}^{-1}\right)$. In the triple decker, the $\mathrm{Ce}$ atom moves towards the external ring but the average distance to the two rings is about the same as in the double decker, leading to roughly the same CF. As shown in Section S4.3 (Eq. $\mathrm{S} 27$ ), for the dimer $\Delta$ includes a two-electron term, due to the difference of interactions between $\sigma$ and $\pi$ local orbitals. The third manifold arises from $f_{\sigma} f_{\phi}$ configuration around 800 $\mathrm{cm}^{-1}$ which corresponds to the $f_{\sigma}-f_{\phi}$ energy gap and the fourth manifold arises from $f_{\pi} f_{\pi}$ configuration lying around $2 \Delta$. The energy of the other configurations are given in Table S3. CASPT2 overestimates SF singlet-triplet gap in $f_{\sigma}^{A} f_{\sigma}^{B}$ configuration and alters the ordering of the CF states: CASPT2 results are not reliable for this molecule. A recent work, ${ }^{76}$ issued from a joint preliminary study, reports similar values of $J$, using the geometry of the monomer.
With the inclusion of SOC, the states are grouped and each manifold can be assigned to a $m_{j}^{A} \otimes m_{j}^{\prime}{ }_{j}^{B}$ configuration as shown in Table S3.

\subsubsection{Spin Hamiltonian parameters}

The ground manifold arises from $1 / 2^{A} \otimes 1 / 2^{B}$ and leads to four states

$$
\begin{aligned}
& | \pm \pm\rangle=|\mathcal{D}, \pm\rangle^{A} \otimes|\mathcal{D}, \pm\rangle^{B} \\
& | \pm \mp\rangle=|\mathcal{D}, \pm\rangle^{A} \otimes|\mathcal{D}, \mp\rangle^{B}
\end{aligned}
$$

As shown in Section S4.2, these four states form a pseudo-singlet, with $\mathbb{S}=0$ and corresponding eigenstate $|\mathcal{S}, 0\rangle=\frac{1}{\sqrt{2}}(|+-\rangle-|-+\rangle)$ and a pseudo-triplet, with $\mathbb{S}=1$ and corresponding eigenstates $|\mathcal{T}, \pm 1\rangle=| \pm \pm\rangle$ and $|\mathcal{T}, 0\rangle=$ $\frac{1}{\sqrt{2}}(|+-\rangle+|-+\rangle)$. The pseudo-spin kets fulfill the following property $\hat{\mathbb{S}}_{z}|\mathbb{S}, \mathbb{M}\rangle=\mathbb{M}|\mathbb{S}, \mathbb{M}\rangle$. Spin Hamiltonian may be expressed using the total pseudo-spin operator (see Eqs. S16 and S17), omitting the rhombic parameter $E$ for symmetry reasons

$$
\begin{aligned}
\hat{\mathcal{H}}_{S}= & -J \hat{\mathbb{S}}^{2}+\hat{\mathbf{S}} \cdot \mathbf{D} \cdot \hat{\mathbf{S}}+\mu_{B} \boldsymbol{B} \cdot \mathbf{G} \cdot \hat{\mathbf{S}} \\
= & -\frac{1}{2} J \hat{\mathbb{S}}^{2}+\frac{3}{4} J+\frac{1}{2} D\left[\hat{\mathbb{S}}_{z}^{2}-\frac{1}{3} \hat{\mathbb{S}}^{2}\right] \quad(14) \\
& +\mu_{B}\left(B_{x} G_{\perp} \hat{\mathbb{S}}_{x}+B_{y} G_{\perp} \hat{\mathbb{S}}_{y}+B_{z} G_{\|} \hat{\mathbb{S}}_{z}\right)
\end{aligned}
$$

and its matrix representation is:

\begin{tabular}{cccc}
$|\mathcal{S}, 0\rangle$ & $|\mathcal{T}, 1\rangle$ & $|\mathcal{T}, 0\rangle$ & $|\mathcal{T},-1\rangle$ \\
\hline$\frac{3 J}{4}$ & 0 & 0 & 0 \\
0 & $-\frac{J}{4}+\frac{D}{6}+\gamma_{z}$ & $\frac{\gamma_{x}-i \gamma_{y}}{\sqrt{2}}$ & $\frac{E}{2}$ \\
0 & $\frac{\gamma+i \gamma_{y}}{\sqrt{2}}$ & $-\frac{J}{4}-\frac{D}{3}$ & $\frac{\gamma_{x}-i \gamma_{y}}{\sqrt{2}}$ \\
0 & $\frac{E}{2}$ & $\frac{\gamma_{x}+i \gamma_{y}}{\sqrt{2}}$ & $-\frac{J}{4}+\frac{D}{6}+\gamma_{z}$
\end{tabular}

with $\gamma_{u}=\mu_{B} B_{u} G_{u}$. More details are given in Section S4.2. Spin Hamiltonian parameters are deduced from the model space formed by the four low lying SO states; the two zero field parameters $J$ and $D$ are deduced from the SO energies, $E$ vanishes due to $\mathcal{D}_{8 h}$ symmetry and $G_{\|}$ and $G_{\perp}$ are calculated from the three representation matrices of the components of the magnetic moment operators $\hat{m}_{u}(u=x, y, z)$ following a procedure previously applied to $\mathrm{NpCl}_{6}{ }^{2-} .59$ As shown in Section S4.2, this procedure does not permit to determine the signs of $G_{\|}$and $G_{\perp}$. However these signs may be determined by constraining the set of wave functions of the 
Table 4: Energy gaps for the first excited states of $\left[\mathrm{Ce}_{2}(\mathrm{COT})_{3}\right]$ and spin Hamiltonian ( $\left.\mathrm{SH}\right)$ parameters defined in Eq. 15 calculated for the three structures of Table 1. Energies are given in $\mathrm{cm}^{-1}$. For SF results, the states are grouped according to their configuration.

\begin{tabular}{|c|c|c|c|c|c|c|c|c|c|c|c|}
\hline \multirow[b]{3}{*}{ struct. } & \multirow[b]{3}{*}{ meth. } & \multicolumn{4}{|c|}{$\mathrm{SF}$} & \multirow{2}{*}{\multicolumn{2}{|c|}{$\frac{\mathrm{SO}}{f_{\sigma} f_{\sigma}}$}} & \multicolumn{4}{|c|}{$\mathrm{SH}$} \\
\hline & & \multirow{2}{*}{$\begin{array}{l}f_{\sigma} f_{\sigma} \\
\text { S-T } \\
\end{array}$} & \multirow{2}{*}{$\begin{array}{c}f_{\sigma} f_{\pi} \\
(8)^{b}\end{array}$} & \multirow{2}{*}{$\begin{array}{c}f_{\sigma} f_{\phi} \\
(8)\end{array}$} & \multirow{2}{*}{$\begin{array}{c}f_{\pi} f_{\pi} \\
(8)\end{array}$} & & & \multicolumn{4}{|c|}{$f_{\sigma} f_{\sigma}$} \\
\hline & & & & & & $|1,0\rangle^{c}$ & $|1,1\rangle$ & $J$ & $D$ & $G_{\|}$ & $G_{\perp}$ \\
\hline \multirow[t]{5}{*}{$\overline{E \text { EXAFS }}$} & CASCI & 5.5 & $\overline{c 679 \pm 2}$ & $857 \pm 1$ & $1359 \pm 3$ & 2.76 & 2.87 & -2.83 & 0.07 & 1.08 & $\begin{array}{l}-2.34 \\
\end{array}$ \\
\hline & $\mathrm{CAS}+\mathrm{S}$ & 18.7 & $618 \pm 2$ & $783 \pm 7$ & $1232 \pm 3$ & 6.78 & 6.19 & -6.38 & -0.39 & 1.10 & -2.32 \\
\hline & DDCI2 & 19.1 & $619 \pm 2$ & $790 \pm 7$ & $1234 \pm 3$ & 6.75 & 6.23 & -6.40 & -0.34 & 1.10 & -2.31 \\
\hline & DDCI3 & 29.9 & $840 \pm 6$ & - & $1675 \pm 5$ & 16.49 & 16.84 & -16.73 & 0.23 & 1.10 & -2.31 \\
\hline & CASPT2 & 61.4 & & & & & & & & & \\
\hline \multirow[t]{5}{*}{ PT2 } & CASCI & 2.5 & $636 \pm 1$ & $572 \pm 1$ & $1271 \pm 2$ & 1.22 & 1.31 & -1.28 & 0.06 & 1.06 & -2.37 \\
\hline & $\mathrm{CAS}+\mathrm{S}$ & 9.6 & $572 \pm 1$ & $726 \pm 3$ & $1142 \pm 4$ & 3.43 & 2.99 & -3.14 & -0.29 & 1.08 & -2.35 \\
\hline & DDCI2 & 9.9 & $548 \pm 1$ & $732 \pm 3$ & $1094 \pm 4$ & 3.38 & 3.01 & -3.14 & -0.25 & 1.08 & -2.35 \\
\hline & DDCI3 & 15.3 & $752 \pm 3$ & - & $1500 \pm 4$ & 8.33 & 8.34 & -8.34 & 0.00 & 1.08 & -2.35 \\
\hline & CASPT2 & 10.8 & & & & & & & & & \\
\hline \multirow[t]{5}{*}{$B 3 L Y P$} & CASCI & 0.5 & $617 \pm 1$ & $719 \pm 1$ & $1242 \pm 4$ & 0.13 & 0.20 & -0.18 & 0.05 & 1.05 & -2.39 \\
\hline & $\mathrm{CAS}+\mathrm{S}$ & 3.4 & $560 \pm 1$ & $648 \pm 1$ & $1127 \pm 5$ & 1.02 & 0.89 & -0.93 & -0.07 & 1.07 & -2.37 \\
\hline & DDCI2 & 3.5 & $561 \pm 1$ & $654 \pm 1$ & $1027 \pm 5$ & 0.97 & 0.87 & -0.91 & -0.05 & 1.07 & -2.37 \\
\hline & DDCI3 & 5.2 & $709 \pm 1$ & - & $1422 \pm 6$ & 2.41 & 2.57 & -2.52 & 0.11 & 1.07 & -2.37 \\
\hline & CASPT2 & 8.0 & & & & & & & & & \\
\hline $\exp ^{d}$ & & & & & & & & -7.92 & \pm 0.020 & \pm 0.974 & \pm 2.335 \\
\hline
\end{tabular}

$a$ : singlet-triplet gap. $b$ degeneracy of the manifold. $c$ energy of $|\mathbb{S}, M\rangle$ relative to $|0,0\rangle . d$ from [33].

model space to behave under the rotations of the molecular system as the components of a real spin. $77,60,69,72$ We show that, within the limit of a vanishing interaction between the two magnetic centers

$$
\begin{aligned}
G_{\|} & =g_{\|} \\
G_{\perp} & =g_{\perp}
\end{aligned}
$$

and consequently $G_{\|}$and $G_{\perp}$ are positive and negative respectively, as $g_{\|}$and $g_{\perp}$ are. Values of $G_{\|}$and $G_{\perp}$ deduced from RASSI calculations are summarized in Tables 4 and S4. The values show almost no dependency on both structure and correlation as for the monomer (see Tables 2 and S1) and are identical to those of monomer. Those values are in very good agreement with the experimental data. This confirms the local nature of the $g$ values. The orbital and spin contributions, $G_{\|}^{L}=\left\langle m_{Z}^{L}\right\rangle=1.1$, $G_{\perp}^{L}=\left\langle m_{X / Y}^{L}\right\rangle=-3.4, G_{\|}^{S}=2 g_{e}\left\langle m_{Z}^{S}\right\rangle=0.2$ and $G_{\perp}^{S}=2 g_{e}\left\langle m_{\perp}^{S}\right\rangle=1.1$, match with Eqs. 10; while in the parallel direction, orbital and spin contributions are additive, they are opposite in the perpendicular direction. It confirms that the four low lying states of the dimer are correctly described as $|\mathcal{D}, \pm 1 / 2\rangle_{A} \otimes|\mathcal{D}, \pm 1 / 2\rangle_{B}$ with $|\mathcal{D}, 1 / 2\rangle_{A}=a\left|f_{\sigma}^{A}\right\rangle-b\left|\bar{f}_{\pi 1}^{A}\right\rangle$.
Spin Hamiltonian parameters are summarized in Table 4. $J$ is negative in accordance with the positive singlet-triplet gap at the SF level and is roughly between the half and the third of the SF gap. This is easily understood by considering the magnetic coupling through the $f_{\sigma}$ orbitals as dominant. As shown in Section 3.2, the ground KD of the monomer is about half $f_{\sigma}$, half $f_{\pi}$, reducing the antiferromagnetic coupling by a factor of 4 . As the singlet-triplet gap, $|J|$ decreases with distance and increases with correlation. The EXAFS/DDCI3 value is $-16.49 \mathrm{~cm}^{-1}$, which is much larger than the experimental coupling of $-7.9 \mathrm{~cm}^{-1}$ while the CASPT2/DDCI3 value $-8.34 \mathrm{~cm}^{-1}$ is closer to the experimental one. $D$ is positive (which means that $|\mathcal{T}, 0\rangle$ is lower than $|\mathcal{T}, 1\rangle$ ) at CASCI and DDCI3 levels and negative at $\mathrm{CAS}+\mathrm{S}$ and DDCI2 levels. The value of $D$ calculated with EXAFS/DDCI3 $\left(D=0.17 \mathrm{~cm}^{-1}\right)$ is much larger than the experimental one $\left|D_{\|}\right|=0.020 \mathrm{~cm}^{-1}$ while the CASPT2 value almost vanishes. Due to $\mathcal{D}_{8 h}$ symmetry, the rhombic parameter $E$ of Eq. S9 vanishes. Its experimental non zero value must arise from distorsions from this symmetry. $\chi$ and $\chi T$ versus $T$ curves calculated for EXAFS geometry are shown in Figure 4 and for CASPT2 geometry in Figure S2. Since in all cases, the sin- 
glet is the ground state, all $\chi$ vs $T$ curves denote an antiferromagnetic magnetic behavior and the position and sharpness of the maximum follows the value of $J ;^{78}$ the best match to the experimental data is obtained for CASPT2/DDCI3 since $J$ is the closest to the experimental value. $\chi T$ at low temperature decreases when $|J|$ increases, as expected, and the slope of the plateau at room temperature is slightly smaller than the experimental one, as for the monomer. It means that here again, the second order Zeeman interaction is slightly too small, and that the energy of the coupled excited states is slightly too high, as is was for the monomer.

\subsubsection{Model Hamiltonian}

In order to analyze the underlying mechanisms to the magnetic coupling, a model Hamiltonian is built within the space of the $f_{\sigma} f_{\sigma}, f_{\sigma} f_{\pi}$ and $f_{\pi} f_{\pi}$ manifolds as developed in Section S4.3 following the approach of reference 79. Mironov proposed a similar model describing the magnetic interaction in $\mathrm{M}_{2} \mathrm{~L}_{10}$ and $\mathrm{M}_{2} \mathrm{~L}_{11}$ cerium dimers, including the $5 d$ orbitals in the model space, ${ }^{28}$ but without matching the model against $a b$ initio calculations. The model parameters are in our case considered within an effective sense, i.e. they include intrinsically the polarization and the superexchange mechanisms due to orbitals other than the $4 f$ ones. The effect of the ionic configurations, with the two magnetic electrons on the same center (as for example $f_{\sigma}^{A 2}$ ) is included perturbatively, but since SOC and CF are about the same, the former can not be treated using perturbation theory. In the $f_{\sigma}^{A} f_{\sigma}^{B}$ space, $|\mathcal{S}, 0\rangle$ is mapped to $\left|S_{g}^{\sigma \sigma}, 0\right\rangle$ (the spin singlet of symmetry $g$ ), and $|\mathcal{T}, \mathcal{M}\rangle$ to $\left|T_{u}^{\sigma \sigma}, M\right\rangle$ (the $M$ component of spin triplet of symmetry $u$ ). Without SOC, the singlet-triplet energy gap splits in an exchange contribution $K^{\sigma \sigma}$ and a kinetic one $-\frac{4 \beta^{\sigma 2}}{U}$ where $K^{\sigma \sigma}$ and $\beta^{\sigma}$ are the exchange and hopping integrals between $f_{\sigma}^{A}$ and $f_{\sigma}^{B}$ and $U$ is the one site repulsion energy. SOC couples $f_{\sigma}^{A} f_{\sigma}^{B}$ and $f_{\sigma}^{A} f_{\pi}^{B}$ manifolds and the latter to $f_{\pi}^{A} f_{\pi}^{B}$. These two excited configurations bring exchange and kinetic contributions to $J$ and $D$. Each contribution is weighted according to the weight of the given configuration $\omega_{\sigma \sigma}=a^{4}, \omega_{\sigma \pi}=2 a^{2} b^{2}$ and $\omega_{\pi \pi}=b^{4}$ where $a$ and $b$ are the coefficients of the one-electron spin on $\sigma$ and $\pi$ respectively as defined for the monomer in Eq. 9 of Section 3.2. These con-

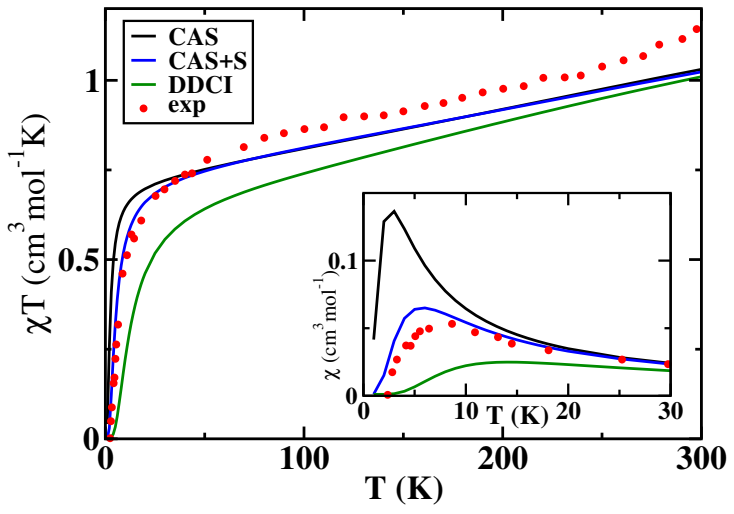

Figure 4: $\chi T$ and susceptibility (inset) vs $T$ for $\left[\mathrm{Ce}_{2}(\mathrm{COT})_{3}\right]$, experimental from [33] and calculated with CAS+CI methods for the EXAFS structure.

tributions, labeled after configuration, exchange $(K)$ and kinetic $(T)$ are derived in Section S4.3

$$
\begin{aligned}
J_{K}^{\sigma \sigma}= & \omega_{\sigma \sigma} K^{\sigma \sigma} \\
J_{T}^{\sigma \sigma}= & -\omega_{\sigma \sigma} \frac{4 \beta^{\sigma 2}}{U} \\
J_{K}^{\sigma \pi}= & \omega_{\sigma \pi}\left[k_{00}^{\sigma \pi}-\frac{1}{3} k_{10}^{\sigma \pi}-\frac{2}{3} k_{11}^{\sigma \pi}\right] \\
J_{T}^{\sigma \pi}= & \omega_{\sigma \pi}\left[-\frac{4 \beta^{\sigma} \beta^{\pi}}{U}\right. \\
& \left.+\frac{K^{U}\left(\beta^{\sigma 2}+10 \beta^{\sigma} \beta^{\pi}+\beta^{\pi 2}\right)}{3 U^{2}}\right] \\
J_{T}^{\pi \pi}= & -\omega_{\pi \pi} \frac{4 \beta^{\pi 2}}{U} \\
D_{K}^{\sigma \pi}= & \frac{2}{3} \omega_{\sigma \pi}\left[k_{11}^{\sigma \pi}-k_{10}^{\sigma \pi}\right]
\end{aligned}
$$

$\beta^{\pi}$ is the hopping integrals between $f_{\pi}^{A}$ and $f_{\pi}^{B}, K^{U}$ is the one-site exchange energy. $f_{\sigma}^{A} f_{\pi}^{B}$ configuration gives rise to two singlets and two triplets which differ by two-electron interactions denoted $k_{S M}^{\sigma \pi}$. The parameters evaluated for the three geometries and the different levels of calculations are given in Tables 5 and S5. The evaluation of the parameters is straightforward with CASCI but for higher levels of correlation, the effective Hamiltonian technique is applied. ${ }^{80,81,82}$ As already pointed out by Calzado et al., one parameter needs to be fixed, and since hopping integrals depend slightly on correlation, we fixed $\beta^{\sigma}$ and $\beta^{\pi}$ to their CASCI value. The different contributions to $J$ and $D$ deduced with Eqs. 17 are given in Tables 5 and S6. Without SOC, $f_{\sigma \sigma}=1$ and $f_{\sigma \pi}=f_{\pi \pi}=0$ and one retrieves the usual coupling scheme 
of two unpaired electrons in two non degenerate orbitals. In all cases, $x=\Delta / \zeta \approx 1$ and $f_{\sigma \sigma}(x) \approx 0.33, f_{\sigma \pi}(x) \approx 0.49$ and $f_{\pi \pi}(x) \approx 0.18$.

- $\beta^{\sigma}$ is large for $4 f$ orbitals separated by more than $4 \AA$ and decreases extremely rapidly with the metal-metal distance. $\beta^{\pi}$ is about a quarter of $\beta^{\sigma}$, due to the smaller overlap between $\pi$ than $\sigma$ orbitals; $\beta^{\pi}$ is still important and leads to non negligible kinetic contributions from the $f_{\sigma}^{A} f_{\pi}^{B}$ manifold.

- The direct exchange $K^{\sigma \sigma}$ decreases rapidly with distance and becomes negative with correlation as already pointed out by Calzado due to spin polarization effects. ${ }^{81}$ At CASCI, $K^{\sigma \sigma}$ is by its very nature an exchange integral and consequently is positive; with correlation, it become effective and its sign undefined. The $\mathrm{CF}$ parameter $\Delta$ is relatively insensitive to distance as it was the case for the monomer, and increases with correlation since it comprises a two-electron term.

- The on-site repulsion $U$ represents the pairing energy of two electrons in the same local $f$ orbitals. As expected, it is independent on metal-metal distance and decreases strongly with correlation, by about $1 / 3$ at $\mathrm{CAS}+\mathrm{S}$ level and by another $1 / 3$ at DDCI3 level.

- The largest contribution to the isotropic magnetic coupling $J$ is $J_{T}^{\sigma \sigma}$ due to the large $\beta^{\sigma}$ value and increases with correlation due to the stabilization of the ionic forms (reduction of $U$ ). With correlation, the parameters are effective, they take into account the physics arising from the outer space and the partition into kinetic and exchange shows a part of arbitrariness. The exchange contribution becomes positive as $K^{\sigma \sigma}$ does. The $f_{\sigma}^{A} f_{\pi}^{B}$ contribution is both kinetic and exchange and may be of both signs.

- The main contribution to the anisotropic coupling is $D_{K}^{\sigma \pi}$ and arises from the differences of two-electron interactions of the different states arising from $f_{\sigma}^{A} f_{\pi}^{B}$ configuration. At $\mathrm{CAS}+\mathrm{S}$ and DDCI2 levels, this contribution is negative while it is positive at CASCI and DDCI3 levels. We can conclude that $D$, whose sign can not be determined from experimental data, is positive, meaning that state $|\mathcal{T}, 0\rangle$ lies lower than the two $|\mathcal{T}, \pm 1\rangle$ components.

The value of $\beta^{\sigma}$ and consequently of $J$ is rather large for a lanthanide dimer and it may be informative to analyze the orbitals participating to the coupling. The Natural Orbitals arising from the average and the difference of the DDCI3 density matrices for the ground SF spin singlet and triplet are represented in Figures 5 and 6 respectively. For the average density matrix, the deviation of the occupation numbers from 2 for the occupied and from 0 for the virtual measures their participation to the correlation. Those relative to the density matrices difference aim to identify the orbitals participating the most to the singlet-triplet gap and consequently to the magnetic coupling. As expected, the magnetic orbitals (with a population close to 1) are the $f_{\sigma}$, the bonding combination being slightly more populated than the anti-bonding one.

- In the space of $\sigma \operatorname{MOs}\left(a_{1 g}\right.$ and $\left.a_{2 u}\right)$, the most participating are virtual. $f_{\sigma u}^{p o l}$ is a polarization orbital for the magnetic orbitals and stabilizes the ionic form, when two electrons are on the same site. The metal $5 d_{\sigma}$ orbitals brings a hybridization allowed by correlation. The largest contribution arises from a $\sigma_{C H}^{*}$ orbital of the bridging ligand which gathers its density in the middle of the cycle, in a zone favoring the overlap of $\sigma$ type metal orbitals. Its participation may be seen as a superexchange mechanism through a MLCT intermediate.

- The space of $\delta$ MOs (symmetries $e_{2 g}, e_{2 u}$ ) consists of combinations of HOMOs of the ligands and of the virtual $f_{\delta}$. Due to symmetry, they can not mix with the magnetic orbitals; they essentially contribute to both spin and charge polarizations leading to the reduction of the effective energy $U$. The same is observed to a lesser extent in the space of $\pi$ MOs with the HOMO- 1 of the ligands and the metal $f_{\pi}$.

- Regarding the natural orbitals of the density matrices difference, there is a one-toone correspondence between orbitals with positive and negative differential occupation numbers: the metal-ligand bonding MOs appearing with a positive number which denotes a larger contribution to the singlet state. 
Table 5: Model parameters for $\left[\mathrm{Ce}_{2}(\mathrm{COT})_{3}\right]$ deduced from CI calculations. All energies in $\mathrm{cm}^{-1}$.

\begin{tabular}{cccccccccccccc}
\hline geom/CI & $\beta^{\sigma}$ & $\beta^{\pi}$ & $K^{\sigma \sigma}$ & $\Delta$ & $U$ & $K^{U}$ & $J_{K}^{\sigma \sigma}$ & $J_{T}^{\sigma \sigma}$ & $J_{K}^{\sigma \pi}$ & $J_{T}^{\sigma \pi}$ & $J_{T}^{\pi \pi}$ & $D_{K}^{\sigma \pi}$ & $D_{T}^{\sigma \pi}$ \\
\hline \hline DFT/CAS & 236 & 50 & 0.7 & 608 & 179005 & 7019 & 0.22 & -0.38 & 0.03 & -0.13 & -0.01 & 0.14 & 0.0003 \\
DFT/CAS+S & 236 & 50 & -0.5 & 558 & 129190 & 10860 & -0.14 & -0.51 & -0.01 & -0.19 & -0.01 & -0.08 & 0.0009 \\
DFT/DDCI3 & 236 & 50 & -1.65 & 691 & 65130 & 3750 & -0.55 & -1.13 & -0.20 & -0.37 & -0.02 & 0.06 & 0.001 \\
\hline PT2/CAS & 447 & 130 & 2.0 & 564 & 176700 & 5520 & 0.63 & -1.44 & 0.09 & -0.66 & -0.07 & 0.22 & 0.0007 \\
PT2/CAS+S & 447 & 130 & 0.0 & 564 & 126430 & 9077 & 0.60 & -1.90 & -0.12 & -0.98 & -0.10 & -0.17 & 0.002 \\
PT2/DDCI3 & 447 & 130 & -2.7 & 727 & 65270 & 3680 & 0.68 & -4.17 & 2.23 & -1.84 & -0.17 & 0.17 & 0.003 \\
\hline EXAFS/CAS & 644 & 180 & 4 & 668 & 175430 & 4730 & 0.35 & -4.40 & 1.01 & -1.92 & -0.19 & 0.31 & 0.002 \\
EXAFS/CAS+S & 644 & 180 & 1.1 & 606 & 123010 & 8461 & 0.34 & -4.20 & 0.41 & -2.00 & -0.20 & -0.12 & 0.005 \\
EXAFS/DDCI3 & 644 & 180 & -3.1 & 800 & 63880 & 4420 & -1.11 & -9.31 & 0.27 & -3.76 & -0.32 & 0.43 & 0.01 \\
\hline
\end{tabular}

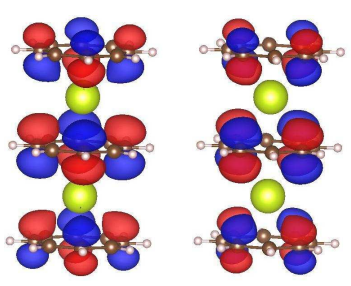

$e_{2 u}: 1 \pi_{2 u}$ 1.9972

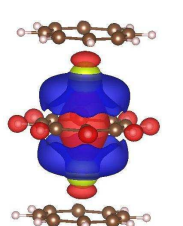

$a_{1 g}: \sigma_{C H}^{*}$

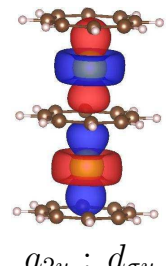

$a_{2 u}: d_{\sigma u}$ 0.0023

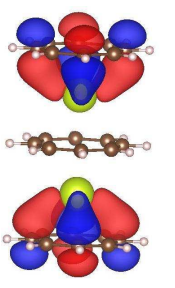

$e_{2 g}: \pi_{2 g}$
1.9979

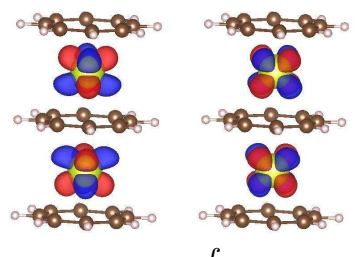

$e_{2 g}: f_{\delta g}$

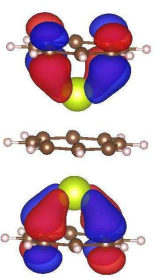

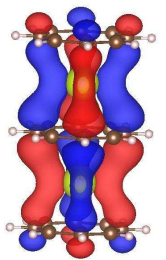

$e_{2 u}: 2 \pi_{2}$
1.9983

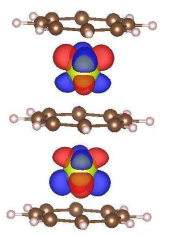

$e_{2 u}: f_{\delta u}$
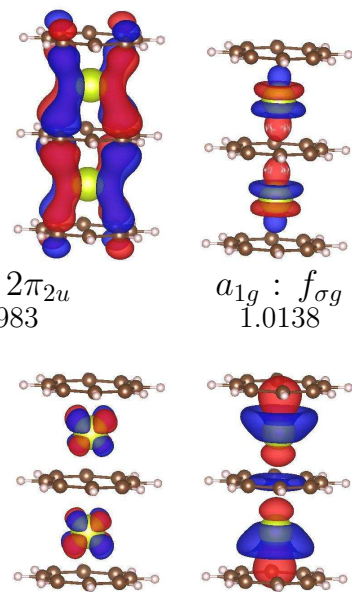

$a_{1 g}: f_{\sigma g}$

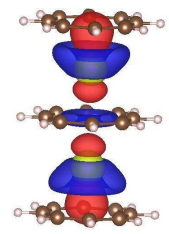

$a_{1 g}: d_{\sigma g}$

0.0017

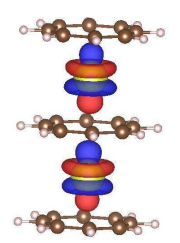

$a_{2 u}: f_{\sigma u}$ 0.9674

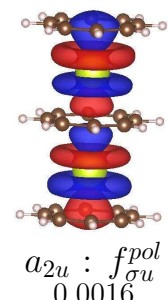

Figure 5: Isosurfaces and occupation of NOs issued from the average of the density matrices of the ground $\mathrm{SF}$ singlet and triplet states at $\mathrm{CAS}(2,6) \mathrm{DDCI} 3$ level for $\left[\mathrm{Ce}_{2}(\mathrm{COT})_{3}\right]$ with structure EXAFS. Isovalue : $\pm 0.03 \mathrm{au}$. 

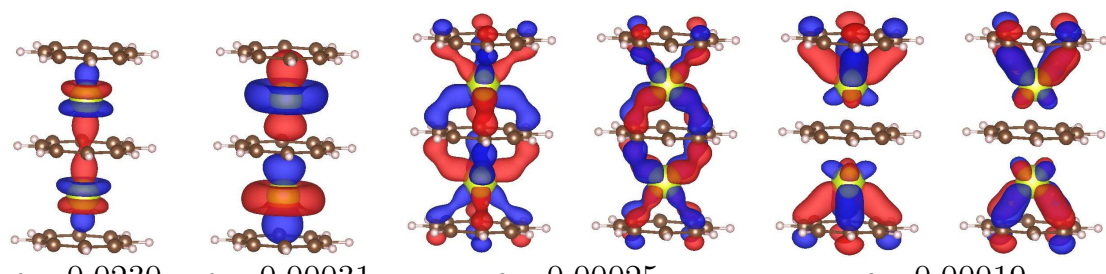

$e_{2 u} 0.00025$

$e_{2 g} 0.00019$

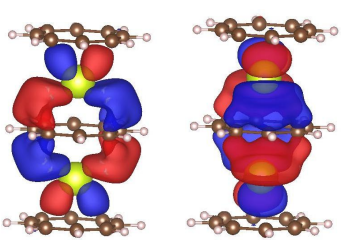

$e_{1 u} 0.00010$

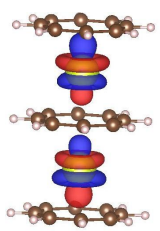

$a_{2 u}-0.0233$

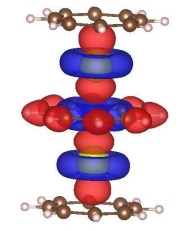

$a_{1 g}-0.00010$

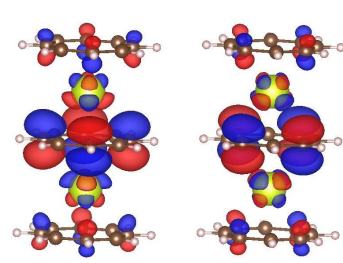

$e_{2 u}-0.00030$

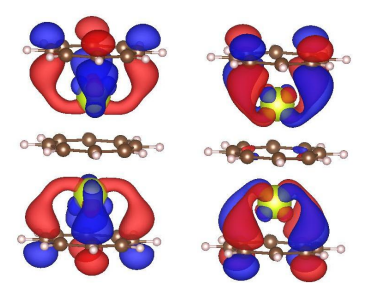

$e_{2 g}-0.00015$

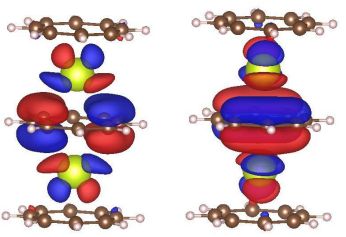

$e_{1 g}-0.00011$

Figure 6: Isosurfaces and occupation of NOs issued from the difference between the density matrices of the ground SF singlet and triplet states calculated at CAS $(2,6)$ DDCI3 for $\left[\mathrm{Ce}_{2}(\mathrm{COT})_{3}\right]$ with structure EXAFS. A positive (negative) occupation denotes a larger participation to the singlet (triplet) state. Isovalue : $\pm 0.03 \mathrm{au}$.
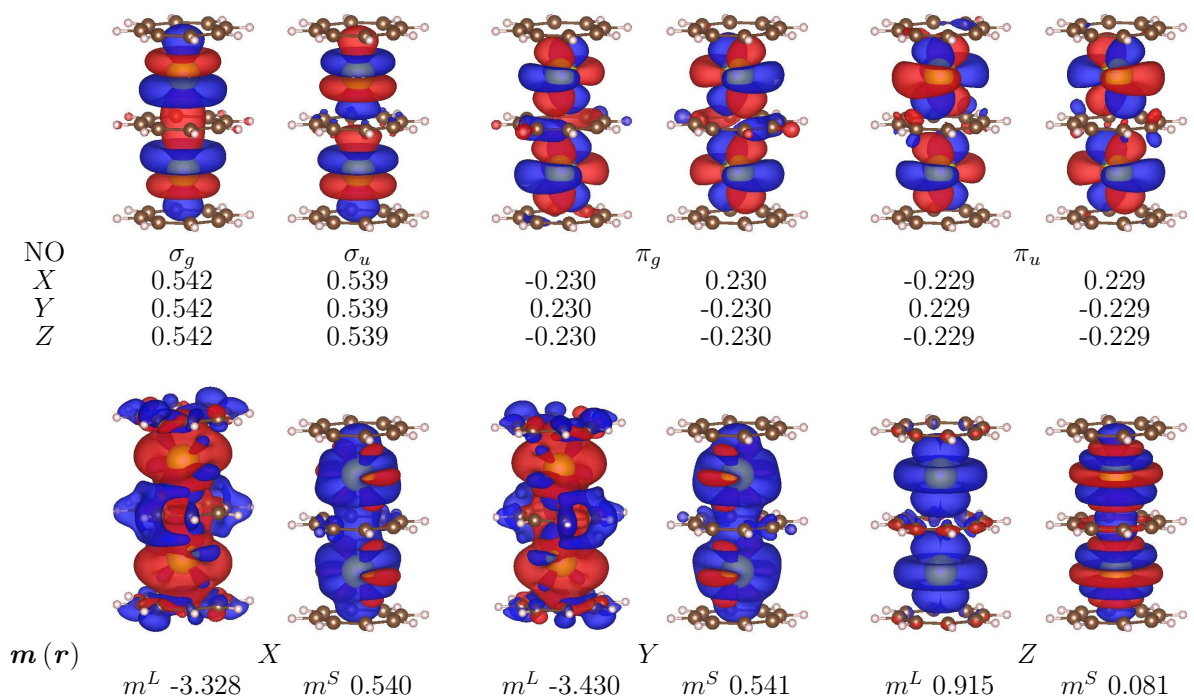

$m^{L}-3.430-m^{S} 0.541$

$m^{L} 0.915$

$m^{S} 0.081$

Figure 7: Isosurfaces and spin occupation of NSOs (top row) and isosurface of orbital and spin magnetizations (bottom row) for a magnetic field in direction $X, Y$ and $Z$ for the ground pseudo-triplet for $\left[\mathrm{Ce}_{2}(\mathrm{COT})_{3}\right]$ with structure EXAFS. Isovalues: \pm 0.005 au for orbitals and \pm 0.00002 for magnetization. $X$ and $Y$ arise from the coupling between $|\mathcal{T}, \pm 1\rangle$ and $|\mathcal{T}, 0\rangle$ while $Z$ arise from the coupling between the two $|\mathcal{T}, \pm 1\rangle$. 
Finally, the NSOs of the pseudo-triplet are depicted in Figure 7. As shown in references 83, 73, the axial magnetization arises from the coupling between the two $|\mathcal{T}, \pm 1\rangle$ while the transverse magnetization arises from the coupling between one of the $|\mathcal{T}, \pm 1\rangle$ with $|\mathcal{T}, 0\rangle$. As shown in Section $\mathrm{S} 4.2$, the $\mathrm{g}$ factors of the pseudo-triplet of the dimer reduces to the $\mathrm{g}$ factors of the pseudodoublet of the monomer in the limit of a vanishing coupling as shown in Eqs. 16. Indeed, the NSOs of the pseudo-triplet of the dimer show the main characteristics of the NSOs of the pseudodoublet of the monomer of Fig. 3. Since for the dimer, they derive from a CAS calculation while from a RAS including the orbitals of the ligand for the monomer, the polarization of the ligands is not as well described as in the monomer. The $\pi_{u}$ NSO shows a small delocalization on the $\pi_{1}$ orbitals of the inner ring and the $\pi_{g}$ NSO a small delocalization on the $\sigma$ system of the inner ring.

\section{Conclusions}

In this work, the cerocene monomer and dimer have been studied using first principle quantum chemistry methods. The high symmetry of the two molecules and the simple electronic structure permit to describe these two molecules using highly correlated methods and to model the magnetic properties with few parameters. For the ground state of the monomer, the singly occupied spinor is the mixing of $f_{\sigma}$ and $f_{\pi}$ spinorbitals with opposite spins and the $\mathrm{g}$ factors are modeled according to the approach proposed by Gourier et al. for $\left[\mathrm{U}\left(\eta^{7}-\mathrm{C}_{7} \mathrm{H}_{7}\right)_{2}\right]^{-}{ }^{68} \mathrm{It}$ is shown that $g_{\|}$is positive while $g_{\perp}$ is negative in order to converge to an isotropic value at the dissociation limit. The $f_{\sigma}-f_{\pi}$ energy gap is about the same as the SOC parameter $\zeta$ and the wave function is close to the $F_{5 / 2}$ term of the free ion. Including the orbitals of the ligands in the active space improves slightly the high temperature susceptibility.

The magnetic coupling between the two Ce(III) ions is relatively important. Since the crystallographic data are not known for the dimer, different structures have been considered. The magnetic coupling has been determined by DDCI3 calculations; it is overestimated for the EXAFS structure and fits the experimental value for the structure optimized with CASPT2. The anisotropic coupling is overestimated by the calculations. The spectrum of the excited states is assigned according to the occupation of the local spinors. The low lying spectrum arises from the coupling of the two local pseudo-doublets giving rise to a pseudo-singlet and a pseudo-triplet. A spin Hamiltonian is written in this model space, either using local pseudo-spin operators or a total spin operator. The $\mathbf{g}$ tensor has been defined within the pseudo-triplet model space and it is shown, that the three $g$ factors of the dimer tend towards those of the monomer, in the limit of non interaction. They are deduced from $a b$ initio calculations and are very close of those of the monomer, confirming that the $\mathbf{g}$ tensor, which models the anisotropy of the magnetic moment is a local property.

In order to get more physical insight in the coupling, a model based on microscopic parameters has been proposed. For a vanishing SOC, one retrieves the Anderson model for the magnetic coupling between two electrons in two non degenerate orbitals. The SOC, by reducing the weight of the wave function on the $\sigma$ orbitals, reduces this $\sigma$ pathway as much but brings further contributions from the $\pi$ pathway. The kinetic contributions are the largest due to large transfer integrals, specially in the $\sigma$ system, but even in the $\pi$ one. The anisotropic coupling arises from the difference between exchange interactions of the different terms arising from $f_{\sigma} f_{\pi}$ configuration. It is shown that in this case, the anisotropy of the coupling is not related to the anisotropy of the local magnetic moments as described by the $g$ factors and that a modelization of the type $J\left(\mathrm{~g}^{A} \cdot \hat{\mathbb{S}}^{A}\right) \cdot\left(\mathrm{g}^{B} \cdot \hat{\mathbb{S}}^{B}\right)$ would not be suitable. This was already pointed out by Van Vleck and Mironov. The two anisotropies are however related since they both arise from the splitting of the ground J manifold by the field of the ligands which partially quenches the orbital moment and determines the nature of the ground Kramers doublets. One could expect the anisotropy of the coupling to arise from the difference of overlap along the $\sigma$ and $\pi$ pathways, but this work shows that this is not the case and that in the present case, the anisotropy of the coupling arises from two-electron interactions.

The large transfer integrals suggest that the $4 f$ orbitals, which are known to have a semi core character and a small overlap with the surrounding, are taken over from other orbitals. The analysis of natural orbitals suggests a superexchange mechanism through a $\sigma_{C H}^{*}$ of the bridging ligand favored by a local hybridization of the 
local $4 f_{\sigma}$ with the virtual $5 d_{\sigma}$ orbitals and an important spin and charge polarization through the HOMOs of the bridging ligand and the virtual $4 f_{\delta}$ orbitals.

\section{Acknowledgments}

Prof. Dr. Frank T. Edelmann (Magdeburg, Germany) is warmly acknowledged for providing the crystallographic data of $\left[\mathrm{Li}(\mathrm{THF})_{4}\right]\left[\mathrm{Ce}(\mathrm{COT})_{2}\right]$ and Dr Nicolas Suaud (Toulouse, France) for providing the code for building the Natural Orbitals from CASDI calculations. This work was supported by the ANR under convention NANR-17-CE06-0010.

\section{Supporting Information Available}

All labels starting with $\mathrm{S}$ refer to Supporting Information. They provide WFT results for $\left[\mathrm{Ce}(\mathrm{COT})_{2}\right]^{-}$and $\left[\mathrm{Ce}_{2}(\mathrm{COT})_{3}\right]$, DFT results for $\left[\mathrm{Ce}_{2}(\mathrm{COT})_{3}\right]$. Model Hamiltonians for the monomer and the dimer are presented as well as Spin Hamiltonian for the dimer. This material is available free of charge via the Internet at http://pubs.acs.org.

\section{References}

[1] Sessoli, R.; Powell, A. K. Strategies towards single molecule magnets based on lanthanide ions. Coord. Chem. Rev. 2009, 253, 2328 2341.

[2] Layfield, R. A.; Murugesu, M., Eds.; Lanthanides and actinides in molecular magnetism; Wiley-VCH: Weinheim, 2015.

[3] Pointillart, F.; Cador, O.; Le Guennic, B.; Ouahab, L. Uncommon lanthanide ions in purely $4 \mathrm{f}$ Single Molecule Magnets. Coord. Chem. Rev. 2017, 346, $150-175$.

[4] Habib, F.; Murugesu, M. Lessons learned from dinuclear lanthanide nano-magnets. Chem. Soc. Rev. 2013, 42, 3278.

[5] Pointillart, F.; Le Guennic, B.; Maury, O.; Golhen, S.; Cador, O.; Ouahab, L. Lanthanide dinuclear complexes involving tetrathiafulvalene- 3-pyridine-N-oxide ligand: Semiconductor radical salt, magnetic, and photophysical studies. Inorg. Chem. 2013, 52,1398 .
[6] Ke, H.; Zhang, S.; Li, X.; Wei, Q.; Xie, G.; Wang, W.; Chen, S. A Dy2 single-molecule magnet with benzoate anions and phenol$\mathrm{O}^{-}$bridging groups. Dalton Trans. 2015, 44, 21025 .

[7] Xue, S. F.; Guo, Y. N.; Ungur, L.; Tang, J. K.; Chibotaru, L. F. Tuning the magnetic interactions and relaxation dynamics of $\mathrm{Dy}_{2}$ SingleMolecule Magnets. Chem. Eur. J. 2015, 21, 14099 .

[8] Wang, Y.-L.; Han, C.-B.; Zhang, Y.-Q.; Liu, Q.-Y.; Liu, C.-M.; Yin, S.-G. Fine-tuning ligand to modulate the magnetic anisotropy in a carboxylate-bridged Dy2 Single-Molecule Magnet system. Inorg. Chem. 2016, 55, 5578.

[9] Zhang, L.; Jung, J.; Zhang, P.; Guo, M.; Zhao, L.; Tang, J.; Le Guennic, B. Site-resolved two-step relaxation process in an asymmetric $\mathrm{Dy}_{2}$ Single-Molecule Magnet. Chem. Eur. J. 2016, 22, 1392.

[10] Zhang, L.; Zhang, Y.-Q.; Zhang, P.; Zhao, L.; Guo, M.; Tang, J. Single-Molecule Magnet behavior enhanced by synergic effect of singleion anisotropy and magnetic interactions. Inorg. Chem. 2017, 56, 7882.

[11] Kan, J.; Wang, H.; Sun, W.; Cao, W.; Tao, J.; Jiang, J. Sandwich-type mixed tetrapyrrole rare-earth triple-decker compounds. Effect of the coordination geometry on the SingleMolecule-Magnet nature. Inorg. Chem. 2013, 52,8505 .

[12] Xiong, H. Y. J.and Ding; Meng, Y. S.; Gao, C.; Zhang, X. J.; Meng, Z. S.; Zhang, Y. Q.; Shi, W.; Wang, B. W.; Gao, S. Hydroxide-bridged five-coordinate DyIII singlemolecule magnet exhibiting the record thermal relaxation barrier of magnetization among lanthanide-only dimers. Chem. Sci. 2017, 8, 1288-1294.

[13] Viciano-Chumillas, M.; Koprowiak, F.; Mutikainen, I.; Wernsdorfer, W.; Mallah, T.; Bolvin, H. Hysteresis in a bimetallic holmium complex: A synergy between electronic and nuclear magnetic interactions. Phys. Rev. B 2017, 96, 214427.

[14] Chow, C. Y.; Bolvin, H.; Campbell, V. E.; Guillot, J. W. R. Kampf; Wernsdorfer, W.; Gendron, F.; Autschbach, J.; Pecoraro, V.; Mallah, T. Assessing the exchange coupling in binuclear lanthanide (III) complexes and the slow relaxation of the magnetization in the antiferromagnetically coupled Dy-2 derivative. Chem. Sci. 2015, 6, 4148. 
[15] Van Vleck, J. H. The theory of electric and magnetic susceptibilities; The Clarendon Press: Oxford, 1932.

[16] Van Vleck, J. H.; Sherman, A. The quantum theory of valence. Rev. Mod. Phys. 1935, 7, $167-228$.

[17] Dirac, P. A. M. Quantum mechanics of manyelectron systems. P. R. Soc. Lond A Mat. 1929, 123, 714-733.

[18] Van Vleck, J. H. Note on the use of the Dirac vector model in magnetic material. Revista de Matemática y Física Teiórica (Tucumián, Argentina) 1962, 14, 189

[19] Anderson, P. W. Theory of magnetic exchange interactions: exchange in insulators and semiconductors. Solid State Physics 1963, 14, 99 214 .

[20] Lukens, W. W.; Walter, M. D. Quantifying exchange coupling in f-ion pairs using the diamagnetic substitution method. Inorg. Chem. 2010, 49, 4458 .

[21] Iwahara, N.; Chibotaru, L. F. Exchange interaction between $J$ multiplets. Phys. Rev. B 2015, 91, 174438.

[22] Lines, M. E. Orbital angular momentum in the theory of paramagnetic clusters. J. Chem. Phys. 1971, 55, 2977.

[23] Chibotaru, L. F.; Ungur, L.; Soncini, A. The origin of nonmagnetic Kramers doublets in the ground state of dysprosium triangles: evidence for a toroidal magnetic moment. Angew. Chem. Int. Ed. 2008, 120, 4194-4197.

[24] Levy, P. M. Rare-earth-iron exchange interaction in the garnets. I. Hamiltonian for anisotropic exchange interaction. Phys. Rev. 1964, 135, A155-A165.

[25] Birgeneau, R. J.; Hutchings, M. T.; Baker, J. M.; Riley, J. D. High-degree electrostatic and exchange interactions in rare-earth compounds. J. Appl. Phys. 1969, $40,1070-1079$.

[26] Santini, P.; Carretta, S.; Amoretti, G.; Caciuffo, R.; Magnani, N.; Lander, G. H. Multipolar interactions in $f$-electron systems: The paradigm of actinide dioxides. Rev. Mod. Phys. 2009, 81, 807-863.

[27] Chibotaru, L. F.; Iwahara, N. Ising exchange interaction in lanthanides and actinides. New J. Phys. 2015, 17, 103028.
[28] Mironov, V. S. Superexchange interaction between lanthanide $\mathrm{f}^{1}$ ions. Spin Hamiltonian calculations for the $90^{\circ}$ and $180^{\circ} \mathrm{f}^{1}-\mathrm{f}^{1}$ superexchange. J. Phys.:Condens. Matter. 1996, 8, 10551.

[29] Moreno Pineda, E.; Chilton, N. F.; Marx, R.; Dörfel, M.; Sells, D. O.; Neugebauer, P.; Jiang, S. D.; Collison, D.; van Slageren, J.; McInnes, E.; Winpenny, R. E. P. Direct measurement of dysprosium(III)...dysprosium(III) interactions in a single-molecule magnet. Nat. Commun. 2014, 5, 5243.

[30] Giansiracusa, M. J.; Moreno-Pineda, E.; Hussain, R.; Marx, R.; Mart Ãnez Prada, M.; Neugebauer, P.; Al-Badran, S.; Collison, D.; Tuna, F.; van Slageren, J.; Carretta, S.; Guidi, T.; McInnes, E. J. L.; Winpenny, R. E. P.; Chilton, N. F. Measurement of Magnetic Exchange in Asymmetric Lanthanide Dimetallics: Toward a Transferable Theoretical Framework. J. Am. Chem. Soc. 2018, 140, 2504-2513.

[31] Hodgson, K.; Mares, F.; Starks, D. F.; Streitwieser, J. A. Lanthanide(III) complexes with cyclooctatrene dianion. Synthetic chemistry, characterization and physical properties.. J. Am. Chem. Soc. 1973, 95, 8650.

[32] Rösch, N.; Streitwieser, A. J. Quasirelativistic SCF-Xalpha scattered wave study of uranocene, thorocene, and cerocene. J. Am. Chem. Soc. 1983, 105, 7237.

[33] Walter, M. D.; Booth, C. H.; Lukens, W. W.; Andersen, R. A. Cerocene revisited: the electronic structure of and interconversion between $\mathrm{Ce}_{2}\left(\mathrm{C}_{8} \mathrm{H}_{8}\right)_{3}$ and $\mathrm{Ce}\left(\mathrm{C}_{8} \mathrm{H}_{8}\right)_{2}$. Organometallics 2009, 28, 698.

[34] Kerridge, A.; Coates, R.; Kaltsoyannis, N. Is cerocene really a $\mathrm{Ce}(\mathrm{III})$ compound ? All electron spin-orbit coupled CASPT2 calculations on $\mathrm{M}($ eta8-C8H8)2 ( $\mathrm{M}=\mathrm{Th}, \mathrm{Pa}, \mathrm{Ce})$. J. Phys. Chem. A 2009, 113, 2896.

[35] Le Roy, J. J.; Jeletic, M.; Gorelsky, S. I.; Korobkov, I.; Ungur, L.; Chibotaru, L.; Murugesu, M. An organometallic building block approach to produce a multidecker $4 \mathrm{f}$ single molecule magnet. J. Am. Chem. Soc. 2013, 135,3502 .

[36] Goodwin, C. A. P.; Ortu, F.; Reta, D.; Chilton, N. F.; Millsi, D. P. Molecular magnetic hysteresis at 60 kelvin in dysprosocenium. Nature 2013, 548, 439-442. 
[37] Le Roy, J. J.; Ungur, L.; Korobkov, I.; Chibotaru, L.; Murugesu, M. Coupling Strategies to Enhance Single-Molecule Magnet Properties of Erbium-Cyclooctatetraenyl Complexes. J. Am. Chem. Soc. 2014, 136, 8003.

[38] Meihaus, K. R.; Long, J. R. Magnetic blocking at $10 \mathrm{~K}$ and a dipolar mediated avalanche in salts of the bis(eta8-cyclooctatretraenide) complex [Er(COT)2]-. J. Am. Chem. Soc. 2013, 135, 17952.

[39] Lueken, H.; Hannibal, P.; Handrick, K. Exchange interactions in lanthanide binuclear compounds. The cubic isotropic case. Chem. Phys. 1990, 143, 151 - 161.

[40] Maurice, R.; Sivalingam, K.; Ganyushin, D.; Guihéry, N.; de Graaf, C.; Neese, F. Theoretical Determination of the Zero-Field Splitting in Copper Acetate Monohydrate. Inorg. Chem. 2011, 50, 6229-6236.

[41] te Velde, G.; Bickelhaupt, F. M.; Baerends, E. J.; van Gisbergen, S. J. A.; Fonseca Guerra, C.; Snijders, J. G.; Ziegler, T. Chemistry with ADF. J. Comput. Chem. 2001, 22, 931-967.

[42] Fonseca Guerra, C.; Snijders, J. G.; te Velde, G.; Baerends, E. J. Towards an order-N DFT method. Theor. Chem. Acc. 1998, 99, 391 .

[43] Baerends, E. J.; Ziegler, T.; Autschbach, J.; Bashford, D.; Bérces, A.; Bickelhaupt, F. M.; Bo, C.; Boerrigter, P. M.; Cavallo, L.; Chong, D. P.; Deng, L.; Dickson, R. M.; Ellis, D. E.; van Faassen, M.; Fan, L.; Fischer, T. H.; Fonseca Guerra, C.; Ghysels, A.; Giammona, A.; van Gisbergen, S. J. A.; Götz, A. W.; Groeneveld, J. A.; Gritsenko, O. V.; Grüning, M.; Gusarov, S.; Harris, F. E.; van den Hoek, P.; Jacob, C. R.; Jacobsen, H.; Jensen, L.; Kaminski, J. W.; van Kessel, G.; Kootstra, F.; Kovalenko, A.; Krykunov, M. V.; van Lenthe, E.; McCormack, D. A.; Michalak, A.; Mitoraj, M.; Neugebauer, J.; Nicu, V. P.; Noodleman, L.; Osinga, V. P.; Patchkovskii, S.; Philipsen, P. H. T.; Post, D.; Pye, C. C.; Ravenek, W.; Rodríguez, J. I.; Ros, P.; Schipper, P. R. T.; Schreckenbach, G.; Seldenthuis, J. S.; Seth, M.; Snijders, J. G.; Solà, M.; Swart, M.; Swerhone, D.; te Velde, G.; Vernooijs, P.; Versluis, L.; Visscher, L.; Visser, O.; Wang, F.; Wesolowski, T. A.; van Wezenbeek, E. M.; Wiesenekker, G.; Wolff, S. K.; K., W. T.; Yakovlev, A. L. Amsterdam Density Functional
[44] van Lenthe, E.; Baerends, E. J.; Snijders, J. G. Relativistic regular two-component Hamiltonians. J. Chem. Phys. 1993, 99, 4597-4610.

[45] Becke, A. Density-functional thermochemistry. III. The role of exact exchange. J. Chem. Phys. 1993, 98, 5648-5652.

[46] Perdew, J. P.; Burke, K.; Ernzerhof, M. Generalized gradient approximation made simple. Phys. Rev. Lett. 1996, 77, 3865-3868.

[47] Perdew, J. P.; Burke, K.; Ernzerhof, M. Generalized gradient approximation made simple [Phys. Rev. Lett. 77, 3865 (1996)]. Phys. Rev. Lett. 1997, 78, 1396.

[48] Ernzerhof, M.; Scuseria, G. E. Assessment of the Perdew-Burke-Ernzerhof exchangecorrelation functional. J. Chem. Phys. 1999, 110, 5029-5036.

[49] Adamo, C.; Barone, V. Toward reliable density functional methods without adjustable parameters: The PBE0 model. J. Chem. Phys. 1999, 110, 6158-6170.

[50] Noodleman, L. Valence bond description of antiferromagnetic coupling in transition metal dimers. J. Chem. Phys. 1981, 74, 5737.

[51] Aquilante, F.; De Vico, L.; Ferré, N.; Ghigo, G.; Malmqvist, P.-A.; Neogrády, P.; Pedersen, T. B.; Pitonak, M.; Reiher, M.; Roos, B.; Serrano-Andrés, M.; Urban, M.; Veryazov, V.; Lindh, R. MOLCAS 7: The next generation. J. Comput. Chem. 2010, 31, 224.

[52] Roos, B. O.; Lindh, R.; Malmqvist, P. A.; Veryazov, V.; Widmark, P. O. Main group atoms and dimers studied with a new relativistic ANO basis set. J. Phys. Chem. A 2004, 108, 2851.

[53] Roos, B. O.; Lindh, R.; Malmqvist, P. A.; Veryazov, V.; Widmark, P. O. New relativistic ANO basis sets for actinide atoms. Chem. Phys. Lett. 2005, 409, 295.

[54] Roos, B. O.; Taylor, P. R.; Siegbahn, P. E. M. A complete active space SCF method (CASSCF) using a density matrix formulated super-CI approach. Chem. Phys. 1980, 48, 157.

[55] Andersson, K.; Malmqvist, P.-A.; Roos, B. O.; Sadlej, A. J.; Wolinski, K. Second-order perturbation theory with a CASSCF reference function. J. Phys. Chem. 1990, 94, 5483.

[56] Malmqvist, P.-A.; Roos, B. O.; Schimmelpfennig, B. The restricted active space (RAS) state interaction approach with spin-orbit coupling. Chem. Phys. Lett. 2002, 357, 230. 
[57] Hess, B. A. Relativistic electronic-structure calculations employing a two-component no-pair formalism with external-field projection operators. Phys. Rev. A 1986, 33, 3742.

[58] Hess, B. A.; Marian, C. M.; Wahlgren, U.; Gropen, O. A mean-field spin-orbit method applicable to correlated wavefunctions. Chem. Phys. Lett. 1996, 251, 365.

[59] Páez Hernàndez, D.; Bolvin, H. Magnetic properties of a fourfold degenerate state: $\mathrm{Np}^{4+}$ ion diluted in $\mathrm{Cs}_{2} \mathrm{ZrCl}_{6}$ crystal. J. Electron. Spectrosc. Relat. Phenom. 2014, 194, 74.

[60] Bolvin, H.; Autschbach, J. Handbook of relativistic quantum chemistry. In ; Liu, W., Ed.; Springer: Berlin, 2017; Chapter Relativistic methods for calculating Electron Paramagnetic Resonance (EPR) parameters.

[61] Bolvin, H. An alternative approach to the g-matrix: theory and applications. ChemPhysChem 2006, 7, 1575.

[62] Benamor, N.; Maynau, D. Size-consistent self-consistent configuration interaction from a complete active space.. Chem. Phys. Lett. 1998, 286, 211.

[63] Miralles, J.; Daudey, J.-P.; Caballol, R. Variational calculation of small energy differences. The singlet-triplet gap in $\left[\mathrm{Cu}_{2} \mathrm{Cl}_{6}\right]^{2-}$. Chem. Phys. Lett. 1992, 198, 555.

[64] Hodgson, K.; Raymond, K. N. Ion pair complex formed between bis(cyclooctatetraenyl)cerium(III) anion and an ether-coordinated potassium cation. Crystal and molecular structure of $\left[\mathrm{K}\left(\left(\mathrm{CH}_{3} \mathrm{CH}_{2} \mathrm{CH}_{2}\right)_{2}\right)\right]\left[\mathrm{Ce}\left(\mathrm{C}_{8} \mathrm{H}_{8}\right)_{2}\right]$. Inorg. Chem. 1972, 11, 3030.

[65] Kilimann, U.; Schaefer, M.; Herbst-Irmer, R.; Edelman, F. T. Cyclooctatetraenyl-komplexe der frühen übergangsmetalle und lanthanoide: IV. Strukturchemie des anionisichen sandwichkomplexes $\left[\mathrm{Ce}(\mathrm{COT})_{2}\right]^{-}$. J. Organomet. Chem. 1994, 469, C15.

[66] Ferraro, F.; Aparecida Barboza, C.; ArratiaPérez, R. A relativistic study of the electronic and magnetic properties of cerocene and thorocene and its anions. J. Phys. Chem. A 2012, 116, 4170 .

[67] Warren, K. D. Ligand field theory of metal sandwich complexes. Magnetic properties of $\mathrm{f}^{x}$ configurations. Inorg. Chem. 1975, 14, 3095.

[68] Gourier, D.; Caurant, D.; Arliguie, T.; Ephritikhine, M. EPR and angle-selected ENDOR study of 5f-ligand interactions in the $\left[\mathrm{U}\left(\eta^{7}\right.\right.$ $\left.\left.\mathrm{C}_{7} \mathrm{H}_{7}\right)_{2}\right]^{-}$anion, an $\mathrm{f}^{1}$ analogue of uranocene. J. Am. Chem. Soc. 1998, 120, 6084.

[69] Notter, F. P.; Bolvin, H. Optical and magnetic properties of the $5 \mathrm{f}^{1} \mathrm{AnX}_{6}^{q-}$ series: a theoretical study. J. Chem. Phys. 2009, 130, 184310.

[70] Gendron, F.; Pritchard, B.; Bolvin, H.; Autschbach, J. Single-ion 4f element magnetism: An ab-initio look at $[\operatorname{Ln}(\mathrm{COT})] 2$-. Dalton Trans. 2015, 19886.

[71] Alessandri, R.; Zulfikri, H.; Autschbach, J.; Bolvin, H. Crystal field in rare-earth complexes: from electrostatics to bonding. Chem. Eur. J. 2018, 24, 5538-5550.

[72] Gendron, F.; Páez Hernàndez, D.; Notter, F. P.; Pritchard, B.; Bolvin, H.; Autschbach, J. Magnetic properties and electronic structure of neptunyl(VI) complexes: Wavefunctions, orbitals, and crystal-field models.. Chem. Eur. J. 2014, 20, 7994.

[73] Gendron, F.; Bolvin, H.; Autschbach, J. Topics in Organometallic Chemistry : Organometallic magnets. In , Vol. 6?; Pointillart, F.; Chandrasekhar, V., Eds.; Springer: , 2018; Chapter Complete Active Space (CAS) wavefunctionbased analysis of magnetization and electronic structure.

[74] Nishino, M.; Yoshioka, Y.; Yamaguchi, K. Effective exchange interactions and magnetic phase transition temperatures in Prussian blue analogs: a study by density functional theory. Chem. Phys. Lett. 1998, 297, 51-59.

[75] Ruiz, E.; Alvarez, S.; Rodriguez-Fortea, A.; Alemany, P.; Pouillon, Y.; Massobrio, C. Magnetism: molecules to materials II. In ; Miller, J. S.; Drillon, M., Eds.; Wiley-VCH: Weinheim, 2001; Chapter Electronic structure and magnetic behavior in polynuclear transition-metal compound.

[76] Solis-Céspedes, E.; MontenegroPohlhammer, N.; Páez-Hernández, D. Theoretical insight into the superexchange mechanism of coupling in f1-f1 system. The case of study $\mathrm{Ce} 2(\mathrm{COT}) 3$ compound. Inorg. Chim. Acta 2018, 477, $192-198$.

[77] Chibotaru, L.; Ceulemans, A.; Bolvin, H. The unique definition of the $\mathrm{g}$ tensor of a Kramers doublet. Phys. Rev. Lett. 2008, 101, 033003.

[78] Kahn, O. Molecular magnetism; Wiley-VCH: New-York, 1993. 
[79] Rota, J. B.; Knecht, S.; Fleig, T.; Ganyushin, D.; Saue, T.; Neese, F.; Bolvin, H. Zero-field splitting of the chalcogen diatomics using relativisitic correlated wave-function methods. J. Chem. Phys. 2011, 114106.

[80] Bloch, B. Sur la théorie des perturbations des états liés. Nucl. Phys. 1958, 6, 329-347.

[81] Calzado, C. J.; Cabrero, J.; Malrieu, J. P.; Caballol, R. Analysis of the magnetic coupling in binuclear complexes. II. Derivation of valence effective Hamiltonians from ab initio CI and DFT calculations. J. Chem. Phys. 2002, 116, 3985-4000.

[82] Bolvin, H. From ab initio calculations to model Hamiltonians: the effective Hamiltonian technique as an efficient tool to describe mixedvalence molecules.. J. Phys. Chem. A 2003, 107,5071 .

[83] Charron, G.; Malkin, E.; Rogez, G.; Batchelor, L. J.; Mazerat, S.; Guillot, R.; Guihéry, N.; Barra, A. L.; Mallah, T.; ; Bolvin, H. Unraveling sigma and pi effects on magnetic anisotropy in cis-NiA 4 B 2 complexes: magnetization, HF-HFEPR studies, first-principles calculations, and orbital modeling. Chem. Eur. J. 2016, 22, 16848-16860. 